\title{
Macroinvertebrados bentónicosy ensayos toxicológicos para evaluar la calidad del agua y del sedimento del río Rímac, Lima, Perú
}

\author{
Benthic macroinvertebrates and toxicological tests for assessing water and \\ sediment quality of the Rimac river, Lima, Peru
}

\author{
Gissela Pascual $^{1}$, José Iannacone ${ }^{1,2,3}$, Lorena Alvariño ${ }^{1}$
}

\section{Resumen}

\begin{abstract}
El objetivo del estudio fue emplear a los macroinvertebrados bentónicos (MIB) y a los ensayos toxicológicos para evaluar en época de avenida y estiaje la calidad del agua y sedimentos del río Rímac (Lima-Perú) durante 2009. Se establecieron doce estaciones de muestreo $\left(\mathrm{E}_{1}-\mathrm{E}_{12}\right)$, teniendo en cuenta las fuentes de contaminación. En cada estación se midieron datos físicos y químicos, MIB, y toxicidad de aguas y sedimentos con Daphnia magna y Chironomus calligraphus. La época de avenida presentó mayores valores de $\mathrm{pH}$. En cambio, mayores valores de conductividad específica, sólidos totales, oxígeno disuelto y transparencia se observaron en la época de estiaje. Entre épocas de avenida y estiaje se observaron diferencias en la abundancia de Planorbidae, Baetidae, Chironomidae, Empididae y Tipulidae. Los índices de diversidad y equitabilidad fueron mayores en época de avenida, pero los índices de dominancia y abundancia fueron mayores en época de estiaje. En el dendrograma de similaridad con base a los MIB se muestra la formación de tres grupos que estarían relacionados a las actividades que se desarrollan en la cuenca en la época de avenida: el primer grupo respuesta a los pasivos mineros, el segundo grupo respuesta a impacto doméstico y agricultura, y el tercer grupo respuesta al impacto por la agricultura, industrias y doméstico. La calidad del agua fue estimada con los índices bióticos, EPT (Ephemeroptera - Plecoptera - Trichoptera) (mayormente mala sobre el 75\%) y el ABI (Andean Biotic Index) (pésimo-mala: 16.67-33.33\%). Para los ensayos toxicológicos con D. magna y con C. calligraphus solo la estación de muestreo $\mathrm{E}_{1}$ fue tóxica en la época de avenida.
\end{abstract}

Palabras clave: calidad de agua; Chironomus; Daphnia; índices bióticos; índices de diversidad

${ }^{1}$ Laboratorio de Ecología y Biodiversidad Animal, Facultad de Ciencias Naturales y Matemática, Universidad Nacional Federico Villarreal, Lima, Perú

${ }^{2}$ Facultad de Ciencias Biológicas, Universidad Ricardo Palma, Lima, Perú

${ }^{3}$ Facultad de Ciencias Ambientales, Universidad Científica del Sur, Lima, Perú

${ }^{4}$ E-mail: joseiannacone@gmail.com

Recibido: 23 de enero de 2019

Aceptado para publicación: 30 de septiembre de 2019 


\section{Abstract}

The aim of this research was to use the benthic macroinvertebrates (MIB) and toxicological assessment tests to evaluate the water and sediment quality of the Rimac river (Lima-Peru) in wet and dry seasons in 2009. Twelve sampling stations $\left(\mathrm{E}_{1}\right.$ to $\left.\mathrm{E}_{12}\right)$ were established, considering the sources of contamination. At each station, physical and chemical data, MIB, and water and sediment toxicity with Daphnia magna and Chironomus calligraphus were measured. The wet season had higher $\mathrm{pH}$; however, higher values of specific conductivity, total solids, dissolved oxygen and transparency were observed in the dry season. Differences in the abundance of Planorbidae, Baetidae, Chironomidae, Tipulidae and Empididae were observed between wet and dry seasons. The diversity and evenness index were higher in wet season, but the dominance and abundance index were higher in the dry season. The dendrogram of similarity based on the MIB showed three groups which would be related to the activities carried out in the basin at the time of flood: the first group responding to mining liabilities, the second group responding to domestic agriculture, and a third group responding to agriculture, industry and domestic impact. Water quality was estimated using the biotic index, EPT (Ephemeroptera - Plecoptera - Trichoptera) (mostly bad about 75\%) and ABI (Andean Biotic Index) (poor - bad: 16.67-33.33\%). For toxicological tests with D. magna and $C$. calligraphus only sampling station $\mathrm{E}_{1}$ was toxic at the time of flood.

Key words: benthic macroinvertebrates; Chironomus; Daphnia magna; water quality

\section{INTRODUCCIÓN}

El río Rímac se encuentra en una de las cuencas hidrográficas más importantes del Perú y es fuente de abastecimiento de agua para consumo humano, actividades industriales, agrícolas, mineras y energéticas. En esta cuenca se encuentran las centrales hidroeléctricas importantes para el Departamento de Lima (Méndez, 2005; Rivera et al., 2007). Sin embargo, sus aguas y sedimentos se encuentran contaminados principalmente por residuos de la industria minero-metalúrgica, desechos industriales y agrícolas, así como por desechos domésticos de los centros poblados ubicados en su entorno (Guillén et al., 1998; Paz-y-Miño et al., 2003; Llontop y Cabrera, 2010), dificultando cada vez más el tratamiento de agua para consumo doméstico.

Se han venido desarrollando diferentes herramientas para evaluar la calidad de los ecosistemas acuáticos, tales como los pará- metros fisicoquímicos del agua según el cuerpo de agua (ríos, lagos, bofedales, mares y otros), los macroinvertebrados bentónicos (MIB) y los bioensayos o ensayos ecotoxicológicos (Iannacone et al., 2003; Muñoz-Riveaux et al., 2003; Trama et al., 2009; Fierro et al., 2012; Villamarín et al., 2013).

Los parámetros fisicoquímicos son muy importantes, pero no proporcionan suficiente información respecto a los efectos de los contaminantes sobre la biota acuática para la protección del medio ambiente dulceacuícola, por lo que es conveniente realizar ensayos toxicológicos (Costa et al., 2008). El principio del WET (por sus siglas en inglés: Whole Effluent Toxicity Testing) consiste en la evaluación del efecto tóxico de un efluente mediante las pruebas de bioensayo (USEPA, 2000). Este tipo de ensayo es utilizado para la evaluación de efluentes, aguas de drenajes o aguas superficiales (SETAC, 2004). Los ensayos toxicológicos estandarizados en el 
laboratorio sobre un determinado modelo biológico sensible como Daphnia magna Müller, 1785 y, Chironomus calligraphus Goeldi, 1905, permiten predecir el riesgo ecológico de muestras de agua y sedimentos, respectivamente (De Schamphelaere y Janssen, 2002; Román-Farje et al., 2017; Alegre et al., 2018).

El análisis fisicoquímico puntual se semejaría a una «fotografía» del río en un momento dado, mientras que el análisis de la comunidad biológica del MIB representaría lo que le ha sucedido durante un periodo determinado como una «película», siendo sensibles a la contaminación orgánica (Rosenberg y Resh, 1993; Muñoz-Riveaux et al., 2003; Alonso y Camargo, 2005; Oller y Goitia, 2005; Figueroa et al., 2007; Iannacone et al., 2013; Rodríguez-Varela et al., 2018). Los MIB están siendo ampliamente empleados en biomonitoreos para la evaluación de la calidad del agua a nivel mundial, debido al amplio espectro de respuestas a perturbaciones del ambiente, taxonomía bien conocida a nivel de familia, disponibilidad de herramientas para el análisis de datos, sensibilidad, sedentarismo, amplia distribución geográfica, y muestreo sencillo y de bajo costo (Figueroa et al., 2003; Guerrero-Bolaño et al., 2003; Alonso y Camargo, 2005; Paredes et al., 2005; Espinoza y Morales, 2008; Rico et al., 2009). Así mismo, se han venido empleando índices bióticos basados en la sensibilidad de diferentes taxones de los MIB a la contaminación por materia orgánica, actividades mineras, eutroficación y perturbaciones antrópicas (Paredes et al., 2004; Alonso y Camargo, 2005; Gonçalves y de Menezes, 2011; Pan et al., 2015).

En el Perú, los estudios con MIB con fines de biomonitoreo de los ecosistemas acuáticos se han incrementado en los últimos años (Iannacone et al., 2003; Paredes et al., 2004, 2005; Acosta et al., 2009; OyaguePassuni, 2009; Ortega et al., 2010; Alván et al., 2012) y vienen siendo considerados como parte de los estudios de evaluación ambiental en los instrumentos de gestión ambiental de las inversiones que comprometen ecosistemas acuáticos, y de igual forma los estudios toxicológicos en ambientes acuáticos con fines de investigación (Arrascue et al., 2001; Iannacone et al., 2005; Iannacone y Salazar, 2007). Sin embargo, aún no se han evaluado los MIB en conjunto con los estudios toxicológicos de agua y sedimentos.

El objetivo de presente estudio fue emplear macroinvertebrados bentónicos y ensayos toxicológicos para evaluar la calidad del agua y del sedimento del río Rímac, LimaPerú, en época de avenida y estiaje. De esta manera se busca contribuir con información básica sobre la calidad ambiental de estas aguas a los trabajos realizados por instituciones y poblaciones interesadas para la mejora de la gestión del río Rímac.

\section{Materiales y Métodos}

\section{Área de Estudio}

El área de estudio corresponde a un sector de la cuenca del río Rímac, Lima-Perú, en el tramo de Bellavista $\left(E_{1}\right)$, distrito de Huarochirí, hasta la captación o bocatoma de agua de la atarjea de SEDAPAL (Servicio de Agua Potable y Alcantarillado de Lima) $\left(\mathrm{E}_{12}\right)$, distrito de El Agustino, con una gradiente altitudinal que va desde 3851 a los $532 \mathrm{msnm}$. Este recorrido tiene aproximadamente $85 \mathrm{~km}$ de longitud (Figura 1).

\section{Muestreos}

Se establecieron doce estaciones de muestreo $\left(E_{1}-E_{12}\right)$ y se realizaron dos muestreos: en época de avenida-verano (febrero-2009) y en estiaje-invierno (junio-2009). La descripción de las estaciones de muestreo se indica en el Cuadro 1.

\section{Parámetros Físicos y Químicos}

En cada estación de muestreo (E) se midió in situ el caudal $\left(\mathrm{L} \cdot \mathrm{m}^{-3}\right)$ por el método de Hynes (1970). El pH, conductividad eléc- 


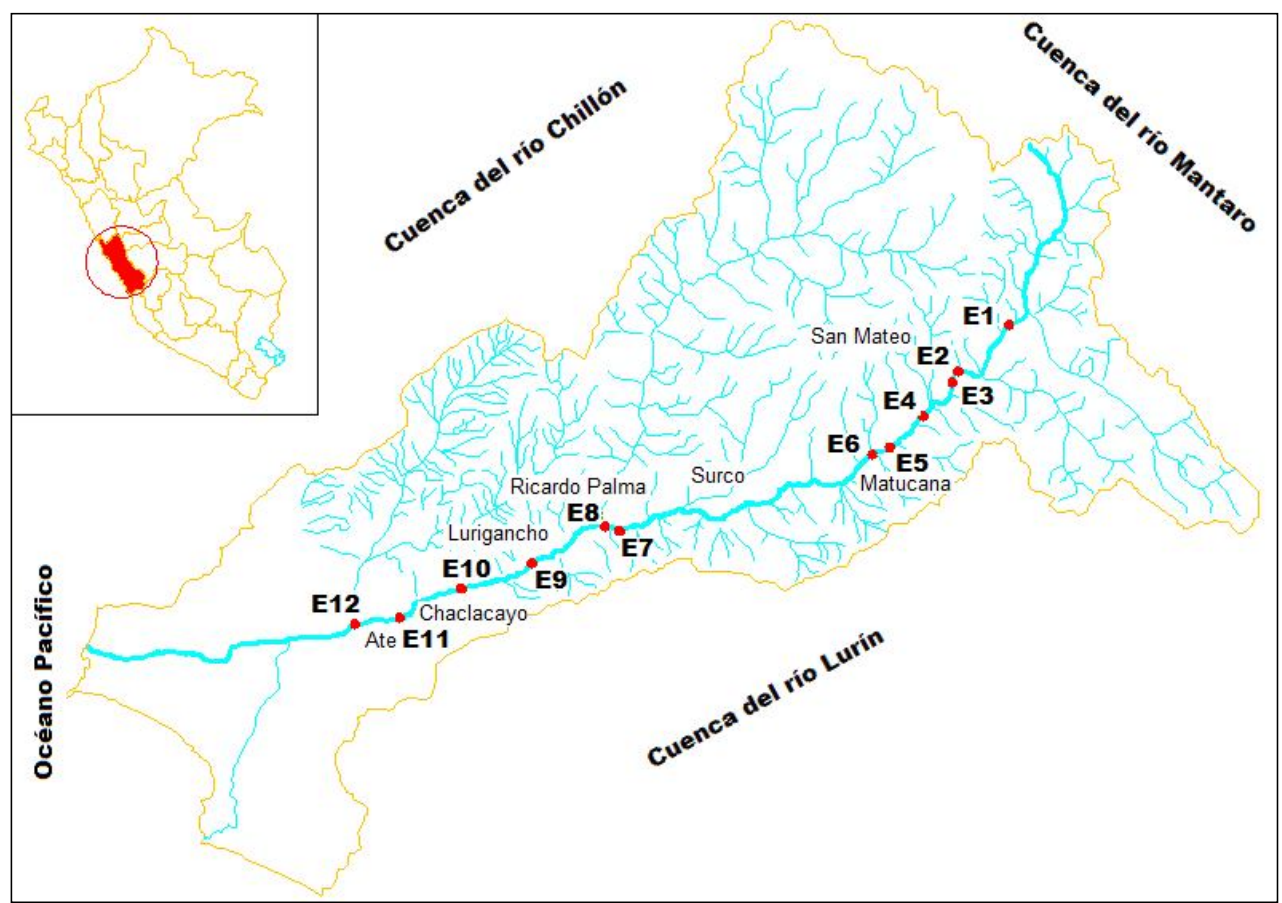

Figura 1. Ubicación de las doce estaciones de muestreo en la cuenca del río Rímac $\left(\mathrm{E}_{1}-\mathrm{E}_{12}\right)$, Lima-Perú

trica (CE, uS), sólidos disueltos totales (SDT, $\left.\mathrm{mg} \cdot \mathrm{L}^{-1}\right)$ y temperatura $\left(\mathrm{T},{ }^{\circ} \mathrm{C}\right)$ fue obtenido con un equipo multiparámetro Hanna ${ }^{\circledR} \mathrm{HI}$ 9811-5. El oxígeno disuelto (OD, $\left.\mathrm{mg} \cdot \mathrm{L}^{-1}\right)$ se calculó por el método de Winkler y la transparencia con el disco de Secchi $(\mathrm{cm})$ (Figueiras y Niell, 1986). En los sitios con alta turbulencia se determinó la trasparencia con base al promedio de un mínimo de tres mediciones. El análisis de la demanda bioquímica de oxígeno $\left(\mathrm{DBO}_{5.20}, \mathrm{mg} \cdot \mathrm{L}^{-1}\right)$ siguió el protocolo señalado por APHA (Abarca, 2007). La granulometría de los sedimentos se determinó por el método de Hynes (1970), considerando seis categorías: arcilla y limo $=<0.06$ $\mathrm{mm}$; arena fina $=0.06-0.25 \mathrm{~mm}$; arena mediana $=0.25-1.0 \mathrm{~mm}$; arena gruesa $=1.00$ $2.00 \mathrm{~mm}$; grava $=2-60 \mathrm{~mm}$; piedra $=60-250$ $\mathrm{mm}$. La profundidad de cada punto de las 12 estaciones no fue evaluada al no encontrarse como parámetro de importancia en los Estándares Nacionales de Calidad de Agua del Perú (DS N. ${ }^{\circ}$ 002-2008-MINAM) ni es considerada en los Índices de Calidad de Agua (ICA) (Bedregal et al., 2010).

Se empleó la prueba de t de Student para comparar los parámetros fisicoquímicos entre la época de avenida y estiaje, y entre las estaciones sobre los $1000 \mathrm{msnm}\left(\mathrm{E}_{1}\right.$ a $\left.\mathrm{E}_{6}\right)$ (ecorregión de la serranía esteparia, cuenca media y alta del río Rímac) y menores a 1000 msnm $\left(E_{7}\right.$ a $\left.E_{12}\right)$ (ecorregión del desierto del Pacífico, cuenca baja del río Rímac) (Bedregal et al., 2010). Los resultados son presentados en promedio \pm error estándar. Para el caso de la granulometría, la categoría arcillo-limo fue catalogada con el valor de 1 y piedras con un valor de 6 , mientras que el resto de las categorías presentaron valores dentro de este rango.

\section{Macroinvertebrados Bentónicos (MIB)}

La colecta en época de avenida y de estiaje se realizó empleando una red Surber- 
modificada por Paredes et al. (2005) de 30 x $30 \mathrm{~cm}$, con $0.25 \mathrm{~mm}$ de abertura de malla (Burrial y Ocharón, 2007). Las muestras triplicadas de MIB fueron recolectadas en sustratos de piedras medianas localizadas en áreas de agua corriente, fijados en alcohol etílico al $80 \%$ y guardados en bolsas y en envases de plástico (Darrigran et al., 2007). En el laboratorio cada muestra biológica fue separada e identificada mayormente a nivel de taxones familia con la ayuda de lupas binoculares, debido a que es un buen indicador de estructura de la comunidad y de las condiciones ambientales (Oscoz et al., 2006; Trama et al., 2009). La identificación de los MIB se realizó empleando las claves taxonómicas especializadas de Domínguez et al. (1992), Merrit y Cummins (1996), Roldán (1998) y Manzo (2005). Los taxones Turbellaria, Nemathelminthes, Oligochaeta y Acarina se identificaron a nivel de taxón superior.

Se calculó el índice de constancia (IC) para determinar la constancia de cada taxón en las dos etapas de muestreo (avenida y estiaje). Los taxones fueron considerados en tres categorías: (1) constantes (C) cuando fueron registrados en más del $50 \%$ de las muestras, (2) comunes (c) cuando se observaron entre un 10 y $50 \%$ de las muestras, y

Cuadro 1. Localización y descripción de las estaciones de muestreo

\begin{tabular}{|c|c|c|c|}
\hline Estación & Coordenadas & $\begin{array}{l}\text { Altitud } \\
(\mathrm{msnm})\end{array}$ & Descripción \\
\hline $\mathrm{E}_{1}$ & $\begin{array}{l}\text { 18L } 0362554- \\
\text { UTM8707648 }\end{array}$ & 3851 & $\begin{array}{l}\text { Rivera amortiguada con vegetación típica de la zona y eucalipto. } \\
\text { Aguas arriba se desarrollan actividades mineras. }\end{array}$ \\
\hline $\mathrm{E}_{2}$ & $\begin{array}{l}\text { 18L0358740 - } \\
\text { UTM8700164 }\end{array}$ & 3831 & $\begin{array}{l}\text { Rivera amortiguada con menos de } 5 \mathrm{~m} \text { de vegetación, aguas arriba se } \\
\text { desarrollan actividades mineras y se encuentra un centro poblado. En } \\
\text { la época seca se vio grasa en el sedimento y olor a desechos fecales. }\end{array}$ \\
\hline $\mathrm{E}_{3}$ & $\begin{array}{l}\text { 18L0357936- } \\
\text { UTM8698630 }\end{array}$ & 3064 & $\begin{array}{l}\text { Rivera amortiguada ampliamente con gramíneas y eucalipto. En los } \\
\text { alrededores se desarrollan actividades agrícolas de pan llevar. }\end{array}$ \\
\hline $\mathrm{E}_{4}$ & $\begin{array}{l}\text { 18L0354175- } \\
\text { UTM8695150 }\end{array}$ & 2676 & $\begin{array}{l}\text { El lado derecho del río se encuentra protegido con un muro de } \\
\text { contención y el ancho de la vegetación amortiguadora está entre 5-10 } \\
\text { m. }\end{array}$ \\
\hline $\mathrm{E}_{5}$ & $\begin{array}{l}\text { 18L0350311- } \\
\text { UTM8691484 }\end{array}$ & 2455 & $\begin{array}{l}\text { Rivera amortiguada ampliamente con gramíneas. Presencia de } \\
\text { residuos sólidos y descarga de aguas residuales domésticas no } \\
\text { tratadas sobre el río. }\end{array}$ \\
\hline $\mathrm{E}_{6}$ & $\begin{array}{l}\text { 18L0348695- } \\
\text { UTM8689910 }\end{array}$ & 2404 & $\begin{array}{l}\text { Presencia de residuos sólidos en las riberas y descarga de aguas } \\
\text { residuales domésticas son tratar. }\end{array}$ \\
\hline $\mathrm{E}_{7}$ & $\begin{array}{l}\text { 18L0318926- } \\
\text { UTM8681454 }\end{array}$ & 971 & $\begin{array}{l}\text { Márgenes del río protegidas con muros de contención, presencia de } \\
\text { residuos sólidos y descarga de aguas residuales domésticas sin tratar. }\end{array}$ \\
\hline $\mathrm{E}_{8}$ & $\begin{array}{l}\text { 18L0317209- } \\
\text { UTM8681292 }\end{array}$ & 926 & $\begin{array}{l}\text { Desarrollo de grandes poblaciones y presencia de residuos sólidos en } \\
\text { las riberas. }\end{array}$ \\
\hline $\mathrm{E}_{9}$ & $\begin{array}{l}\text { 18L0309622- } \\
\text { UTM } 8676342\end{array}$ & 701 & $\begin{array}{l}\text { Descarga de aguas residuales domésticas sobre el río, sin previo } \\
\text { tratamiento y presencia de residuos sólidos. }\end{array}$ \\
\hline $\mathrm{E}_{10}$ & $\begin{array}{l}\text { 18L0300845- } \\
\text { UTM8673904 }\end{array}$ & 532 & $\begin{array}{l}\text { Desarrollo de actividades pecuarias. El río es usado como fuente de } \\
\text { agua para los animales, fines industriales y actividades domésticas } \\
\text { (lavandería). }\end{array}$ \\
\hline $\mathrm{E}_{11}$ & $\begin{array}{l}\text { 18L0292952- } \\
\text { UTM8671074 }\end{array}$ & 601 & $\begin{array}{l}\text { Descarga de aguas residuales domésticas sobre el río, sin previo } \\
\text { tratamiento y presencia de residuos sólidos. Desarrollo de actividades } \\
\text { agrícolas. }\end{array}$ \\
\hline $\mathrm{E}_{12}$ & $\begin{array}{l}\text { 18L0287783- } \\
\text { UTM8670396 }\end{array}$ & 642 & $\begin{array}{l}\text { Aguas arriba hay presencia de residuos sólidos y descarga de aguas } \\
\text { residuales domésticas. Hasta este punto son arrastrados los } \\
\text { contaminantes de aguas arriba. Río abajo se encuentra la planta de } \\
\text { potabilización de agua La Atarjea que abastece a la capital Lima. }\end{array}$ \\
\hline
\end{tabular}


(3) $\operatorname{raros}(r)$ hasta en un 10\% de las muestras (Iannacone y Alvariño, 2007). Los resultados son presentados en promedio \pm error estándar. Se usó la prueba de t de Student para comparar los promedios de abundancia (ind $\cdot \mathrm{m}^{2}$ ) para cada taxón entre épocas (avenida y estiaje) y altitud [entre las estaciones sobre los $1000 \mathrm{msnm}\left(\mathrm{E}_{1} \mathrm{a} \mathrm{E}_{6}\right)$ y menores a $1000 \mathrm{msnm}\left(\mathrm{E}_{7}\right.$ a $\left.\mathrm{E}_{12}\right)$ ]. Se determinaron los siguientes índices comunitarios para las épocas de avenida y estiaje para cada $\mathrm{E}$ de muestreo: Abundancia (ind $\cdot \mathrm{m}^{2}$ ), Riqueza (S), Dominancia de Simpson (D), diversidad de Shannon-Wiener ( $\left.\mathrm{H}^{\prime}\right)$ y Equidad de Pielou (J') (Martella et al., 2012).

Se realizaron dendrogramas para las épocas de avenida y estiaje en base a los MIB con el índice de similaridad cuantitativo de Morisita-Horn empleando el programa estadístico PAST (Palaeontological STatistics v. 1.34) (Hammer et al., 2005). La calidad de las aguas se determinó empleando los siguientes índices bióticos: ABI (Andean Biotic Index) presentado por Acosta et al. (2009) para la evaluación de la calidad ecológica de ríos altoandinos (CERA) y el índice de EPT (Ephemeroptera - Plecoptera - Trichoptera) (Carrera y Fierro, 2001). La aplicación del ABI es similar al BWMP/col (Roldán, 1988, 2003), la cual consiste en identificar los taxones a nivel de familia y asignarles un valor de tolerancia- sensibilidad, luego los valores totales son sumados y se obtiene el valor final asociado a la clase de calidad. Las valoraciones varían entre 0 (taxón tolerante) a 10 (taxón sensible) clasificándolos para el ABI en bueno, moderado, malo y pésimo, y para EPT en muy buena, buena, regular y mala (Figueroa et al., 2003; Muñoz-Riveaux et al., 2003; Alonso y Camargo, 2005; Alomía et al., 2017).

Se usó la prueba de t de Student para comparar los parámetros comunitarios e índices de calidad de agua a base de MIB entre la época de avenida y de estiaje, y entre las seis estaciones sobre los $1000 \mathrm{msnm}$ y las seis menores a $1000 \mathrm{msnm}$. Se determinó mediante el análisis de correspondencia ca- nónica (ACC) la relación entre las variables ambientales y los índices comunitarios, y de calidad de agua con base a los MIB y ecotoxicológicos de la cuenca del río Rímac (Espinoza y Morales, 2008).

\section{Ensayos Ecotoxicológicos}

Se realizaron siguiendo el principio del WET. Para la evaluación del agua se utilizó el microcrustáceo $D$. magna y para el sedimento se empleó a la larva de insecto $C$. calligraphus. La toma de muestras de agua se realizó con un balde de 4 L y los sedimentos en bolsas herméticas de $1 \mathrm{~L}$ de capacidad, que se conservaron en refrigeración hasta el análisis toxicológico (Iannacone et al., 2005; Alegre et al., 2018).

Daphnia magna: Las hembras se obtuvieron de un acuario en el distrito de Lince, LimaPerú. Se realizaron cultivos masivos de $D$. magna en condiciones de laboratorio y se separaron las hembras embrionadas en envases con agua de grifo declorinada mediante aireación. Para el ensayo se utilizaron neonatos con menos de $24 \mathrm{~h}$ de edad. La prueba consistió en un ensayo agudo con cuatro réplicas y lecturas hasta $48 \mathrm{~h}$. Las muestras de agua de río fueron diluidas con agua mineral Cielo®, para el cual se consideraron cinco diluciones: $6.25,12.5,25,50$ y $100 \%$, y un control. Las pruebas se realizaron a $\mathrm{pH}$ $7.3 \pm 0.5$ con temperatura de $21 \pm 2{ }^{\circ} \mathrm{C}$, y las hembras no se alimentaron durante todo el ensayo. La mortalidad se discriminó por la falta de movimiento ante un estímulo externo en 10 segundos de observación (Alegre et al., 2018).

Chironomus calligraphus: Las masas de huevos fueron colectadas en las orillas de las pozas de oxidación de la planta de tratamiento de aguas residuales domésticas (PTAR) de San Juan de Miraflores, Lima-Perú (Iannacone et al., 2001) y cultivadas en condiciones de laboratorio en peceras de vidrio con agua declorinada hasta el segundo estadio larval (Zilli et al., 2008; Canteiro y Albertoni, 2011). Los ensayos se realizaron 
utilizando $80 \mathrm{~g}$ de sedimento colectado a volúmenes de sedimento de $6.25,12.5,25,50 \mathrm{y}$ $100 \%$, y un control, mezcladas con $140 \mathrm{ml}$ de agua embotellada Cielo® y arena fina, previamente desinfectada con hipoclorito de sodio, en donde se expusieron 10 larvas por repetición. Se realizaron cuatro réplicas por concentración. Las pruebas se realizaron a $\mathrm{pH} 7.5 \pm 0.5$ y a una temperatura de $22 \pm$ $2{ }^{\circ} \mathrm{C}$, y durante el ensayo las larvas recibieron $1.5 \mathrm{ml}$ de alimento completo para peces TetraMin ${ }^{\circledR}$ al $3 \%$ en dos oportunidades. Las lecturas se realizaron a los 10 días y se midió la respuesta subletal en la biomasa producida $(\mu \mathrm{g})$ con una balanza analítica. La mortalidad se discriminó por la falta de movimiento por más de $10 \mathrm{~s}$ de observación al microscopio estereoscopio (Iannacone et al., 2000; Alegre et al., 2018).

Las diferencias entre la mortalidad de D. magna y C. calligraphus y en la biomasa producida por $C$. calligraphus entre las concentraciones se evaluaron a través de un análisis de varianza previa transformación al arcoseno de la raíz cuadrada de los datos y con la ayuda del programa TSK se calculó la concentración letal media $\left(\mathrm{CL}_{50}\right)$ a $48 \mathrm{~h}$ para y $\mathrm{CL}_{50}$ a 10 días y los valores de LOEC (concentración más baja de efectos observables) para la biomasa, utilizando el programa estadístico SPSS v. 21.0. Se usó la prueba de t de Student para comparar los ensayos ecotoxicólogicos entre la época de avenida y de estiaje, y entre las seis estaciones sobre los $1000 \mathrm{msnm}$ y las seis menores a $1000 \mathrm{msnm}$.

\section{Resultados}

\section{Parámetros Fisicoquímicos}

El Cuadro 2 muestra las variaciones de parámetros fisicoquímicos de la cuenca del río Rímac en las dos épocas del estudio: avenida-2009 y estiaje-2009. Se observaron durante la época de avenida mayores valores de $\mathrm{pH}$ (avenida $=8.83 \pm 0.10$; estiaje $=$ $7.50 \pm 0.07, \mathrm{t}=10.32 ; \mathrm{p}<0.001)$ y caudal $\left(\mathrm{L} \cdot \mathrm{m}^{-3}\right)$ (avenida $=5.79 \pm 1.01 ;$ estiaje $=1.18 \pm 0.40$, $\mathrm{t}=4.20 ; \mathrm{p}<0.001$ ), $\mathrm{y}$ mayores en CE (uS) (avenida $=414.1 \pm 23.2 ;$ estiaje $=553.9 \pm 25.5$, $\mathrm{t}=4.05 ; \mathrm{p}<0.001), \mathrm{ST}\left(\mathrm{mg} \cdot \mathrm{L}^{-1}\right)($ avenida $=$ $213.2 \pm 13.5$; estiaje $=276.7 \pm 12.5, \mathrm{t}=3.44$; $\mathrm{p}=0.002), \mathrm{OD}\left(\mathrm{mg} \cdot \mathrm{L}^{-1}\right)$ (avenida $=7.40 \pm 0.43$; estiaje $=8.50 \pm 0.20, \mathrm{t}=2.29 ; \mathrm{p}=0.032) \mathrm{y}$ transparencia $(\mathrm{cm})$ (avenida $=10.83 \pm 1.49$; estiaje $=29.41 \pm 4.84, \mathrm{t}=3.66 ; \mathrm{p}<0.001)$ en la época de estiaje. No se encontraron diferencias significativas en la temperatura $\left({ }^{\circ} \mathrm{C}\right)$ (avenida $=$ $17.05 \pm 1.32$; estiaje $=16.32 \pm 1.36, \mathrm{t}=0.38$; $\mathrm{p}=0.70)$ y granulometría (avenida $=$ $4.41 \pm 0.48$; estiaje $=5.25 \pm 0.44, \mathrm{t}=1.26$; $\mathrm{p}=0.21)$. Solo se vieron diferencias altitudinales para la temperatura $\left({ }^{\circ} \mathrm{C}\right)(<1000 \mathrm{msnm}$ $=19.62 \pm 0.67 ;>1000 \mathrm{msnm}=13.75 \pm 1.26$, $\mathrm{t}=4.08 ; \mathrm{p}<0.001$ ), presentándose valores más altos en las seis estaciones menores a 1000 msnm. El resto de los parámetros ambientales no mostraron diferencias altitudinales $(t=0.027-1.97 ; p=0.06-0.97)$.

\section{Macroinvertebrados Bentónicos (MIB)}

Se encontró un total de 23709 MIB, de los cuales 4193 (38 taxones) estuvieron presentes en temporada de avenida y 19516 (30 taxones) en temporada de estiaje, siendo más abundantes los insectos en estadios inmaduros en ambas temporadas. Los órdenes de insectos más abundantes en la temporada de avenida fueron Diptera (32.9\%, 11 familias), Trichoptera $(26.0 \%, 5$ familias $)$, Ephemeroptera (19.5\%, 3 familias) y Coleoptera (19.4\%, 6 familias); mientras que en la temporada de estiaje predominaron en abundancia Diptera (86.5\%, 10 familias) y Ephemeroptera (11.1\%, 3 familias) (Cuadro $3)$. En el cuadro se observa, así mismo, la presencia en las épocas de avenida y estiaje, respectivamente, 8 y 8 taxones constantes, 18 y 11 taxones comunes y 11 taxones raros en ambos casos. Diferencias en la Abundancia entre épocas solo se observó para Planorbidae (avenida $=0.41 \pm 0.19$; estiaje $=$ $0 \pm 0, \mathrm{t}=2.15 ; \mathrm{p}=0.04$ ), Baetidae (avenida $=10.75 \pm 2.30$; estiaje $=160.08 \pm 62.41, \mathrm{t}=2.39$; $\mathrm{p}=0.02$ ), Chironomidae (avenida = 
Cuadro 2. Parámetros fisicoquímicos en época de avenida y estiaje de la cuenca del río Rímac, Lima-Perú (febrero del * Datos no registrados; ** Más profundidad a la cual ya no se

\begin{tabular}{|c|c|c|c|c|c|c|c|c|c|c|c|c|c|c|c|c|c|c|}
\hline & & & & $\begin{array}{r}\mathrm{Av} \\
\text { (febre }\end{array}$ & $\begin{array}{l}\text { enida } \\
\text { o } 200\end{array}$ & & & & & & & & (jun & $\begin{array}{r}\text { Esti } \\
\text { io } 200\end{array}$ & & & & \\
\hline 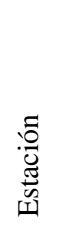 & 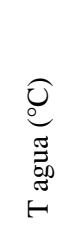 & $\stackrel{I}{2}$ & $\underbrace{\tilde{\Xi}}_{\tilde{U}}$ & 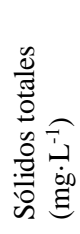 & 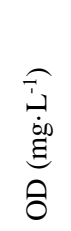 & 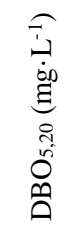 & 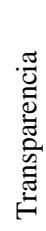 & 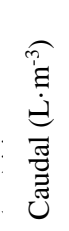 & 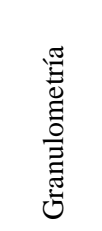 & 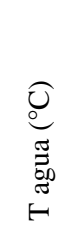 & $\frac{T}{2}$ & 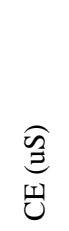 & 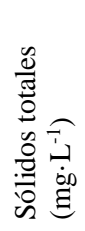 & 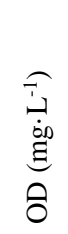 & 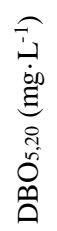 & 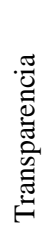 & 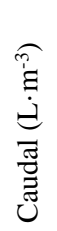 & 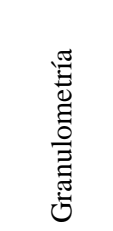 \\
\hline$E_{1}$ & 8.3 & 8.5 & 470 & $*$ & 9.2 & $*$ & 16 & 2.0 & Piedra & 6.3 & 7.7 & 680 & 340 & 7.8 & 1.5 & 6 & 0.2 & Piedra \\
\hline $\mathrm{E}_{2}$ & 10.3 & 8.8 & 334 & 165 & 5.6 & $*$ & 5 & 3.1 & $\begin{array}{l}\text { Arcilla } \\
\text { y limo }\end{array}$ & 8.1 & 7.6 & 433 & 218 & 8.2 & 3.6 & 55 & 1.1 & Piedra \\
\hline $\mathrm{E}_{3}$ & 15.9 & 8.9 & 595 & 300 & 7.6 & $*$ & 25 & 3.3 & Piedra & 14.7 & 7.8 & 518 & 257 & 8.5 & 7.3 & 22 & 0.6 & Piedra \\
\hline $\mathrm{E}_{4}$ & 15.9 & 8.7 & 483 & 239 & 10.6 & 2.61 & 9 & 5.6 & $\begin{array}{l}\text { Arena } \\
\text { gruesa }\end{array}$ & 16.2 & 7.7 & 648 & 322 & 8.9 & 0.5 & 15 & 0.2 & Piedra \\
\hline $\mathrm{E}_{5}$ & 15.5 & 8.2 & 416 & 208 & 6.2 & $*$ & 8 & 3.9 & $\begin{array}{l}\text { Arena } \\
\text { gruesa }\end{array}$ & 18.9 & 7.7 & 537 & 269 & 7.5 & 0.7 & 14 & 0.4 & Piedra \\
\hline $\mathrm{E}_{6}$ & 15.2 & 8.7 & 446 & 214 & 7.9 & $*$ & 10 & 4.8 & $\begin{array}{l}\text { Arena } \\
\text { gruesa }\end{array}$ & 19.7 & 7.8 & 561 & 282 & 7.9 & 7.9 & 24 & 0.6 & Piedra \\
\hline $\mathrm{E}_{7}$ & 20.0 & 9.7 & 453 & 228 & 7.4 & $*$ & 7 & 3.0 & Piedra & 21.2 & 7.4 & 584 & 294 & 10.1 & 5.1 & 55 & 1.0 & Piedra \\
\hline $\mathrm{E}_{8}$ & 18.3 & 8.8 & 342 & 180 & 6.4 & $*$ & 9 & 5.7 & $\begin{array}{l}\text { Arena } \\
\text { gruesa }\end{array}$ & 20.8 & 7.0 & 519 & 263 & 8.6 & 3.6 & 24 & 1.7 & Piedra \\
\hline $\mathrm{E}_{9}$ & 18.2 & 8.7 & 370 & 176 & 8.6 & $*$ & 11 & 13.9 & Piedra & 19.1 & 7.3 & 526 & 263 & 8.6 & 7.4 & 28 & 0.5 & Piedra \\
\hline $\mathrm{E}_{10}$ & 24.0 & 9.2 & 397 & 200 & 5.6 & $*$ & 11 & 5.0 & Piedra & 18.7 & 7.2 & 714 & 352 & 8.1 & 8.1 & 25 & 0.4 & $\begin{array}{l}\text { Arena } \\
\text { gruesa }\end{array}$ \\
\hline$E_{11}$ & 22.0 & 8.9 & 346 & 173 & 7.3 & $*$ & 10 & 8.8 & $\begin{array}{l}\text { Arena } \\
\text { fina }\end{array}$ & 15.9 & 7.4 & 468 & 232 & 9.4 & 5.0 & 30 & 2.4 & $\begin{array}{l}\text { Arena } \\
\text { gruesa }\end{array}$ \\
\hline $\mathrm{E}_{12}$ & 21.0 & 8.9 & 317 & 176 & 6.4 & $*$ & 9 & 10.4 & $\begin{array}{l}\text { Arena } \\
\text { gruesa }\end{array}$ & 16.3 & 7.5 & 459 & 228 & 8.5 & $*$ & 55 & 5.1 & $\begin{array}{c}\text { Arcilla y } \\
\text { limo }\end{array}$ \\
\hline
\end{tabular}

* Datos no registrados; ** Más profundidad a la cual ya no se observó el Disco de Secchi; *** Profundidad máxima a la que se observó el Disco de Secchi; CE = Conductividad eléctrica

$89.50 \pm 56.29 ;$ estiaje $=1361.25 \pm 210.68, \mathrm{t}=$ $5.83 ; \mathrm{p}<0.001$ ), Empididae (avenida $=1.66 \pm 0.60 ;$ estiaje $=14.00 \pm 4.14, \mathrm{t}=2.94$; $\mathrm{p}=0.01)$ y Tipulidae (avenida $=0.41 \pm 0.14$; estiaje $=0 \pm 0, \mathrm{t}=2.80 ; \mathrm{p}=0.02)$. Con relación a la altitud se vio diferencias solo en Baetidae $(<1000 \mathrm{msnm}=153.1 \pm 63.1 ;>1000 \mathrm{msnm}=$ $17.75 \pm 10.25, \mathrm{t}=2.11 ; \mathrm{p}=0.04) \mathrm{y}$ Leptohyphidae $(<1000 \mathrm{msnm}=69.08 \pm 26.77$; $>1000 \mathrm{msnm}=0 \pm 0, \mathrm{t}=2.58 ; \mathrm{p}=0.03)$.

El Cuadro 4 muestra las variaciones de parámetros comunitarios y de calidad de agua de los MIB de la cuenca del río Rímac en las dos épocas en estudio: avenida-2009 y estiaje-2009. Los parámetros comunitarios de Abundancia (avenida $=1294 \pm 563$; estiaje $=$ $6023 \pm 912, \mathrm{t}=4.41 ; \mathrm{p}<0.001)$ y D (avenida $=$ $0.33 \pm 0.03$; estiaje $=0.75 \pm 0.03, \mathrm{t}=7.91$; $\mathrm{p}<0.001)$ fueron mayores en la temporada de estiaje, mientras que $S$ (avenida $=13.41 \pm 1.28$; estiaje $=0 \pm 0, \mathrm{t}=2.03 ; \mathrm{p}=0.04), \mathrm{H}^{\prime}($ avenida $=$ $1.55 \pm 0.12$; estiaje $=0.54 \pm 0.06, \mathrm{t}=7.13$; $\mathrm{p}<0.001)$ y J' (avenida $=0.61 \pm 0.04$; estiaje $=$ $0.23 \pm 0.02, \mathrm{t}=7.13 ; \mathrm{p}<0.001)$ fueron mayores en la temporada de avenida. Con relación a la altitud (msnm) se vio diferencias entre $<1000 \mathrm{msnm}$ y $>1000 \mathrm{msnm}$ solo en $\mathrm{T}$ 
Cuadro 3. Abundancia total (AT) (individuos $\cdot \mathrm{m}^{-2}$ ) de familias de macroinvertebrados bentónicos (MIB) e índices de constancia (IC) durante la época de venida y estiaje en el río Rímac, 2009

\begin{tabular}{|c|c|c|c|c|c|c|c|}
\hline \multirow{2}{*}{ Taxón superior } & \multirow{2}{*}{ Taxón } & \multicolumn{2}{|c|}{ Venida - 2009} & \multicolumn{2}{|c|}{ Estiaje - 2009} & \multirow[b]{2}{*}{ Época } & \multirow[b]{2}{*}{ Altitud } \\
\hline & & $\mathrm{AT}$ & IC & AT & $\mathrm{IC}$ & & \\
\hline Platyhelmintes & Turbellaria* & 4 & $16.67 \mathrm{c}$ & 1 & $8.33 r$ & & \\
\hline Nemathelmintes & Nemathelmintes* & 10 & $41.67 \mathrm{c}$ & 8 & $25.00 \mathrm{c}$ & & \\
\hline Gasteropoda & $\begin{array}{l}\text { Planorbidae } \\
\text { Physidae }\end{array}$ & $\begin{array}{c}5 \\
66\end{array}$ & $\begin{array}{l}33.33 c \\
50.00 c\end{array}$ & $\begin{array}{l}0 \\
2\end{array}$ & $\begin{array}{l}0.00 \\
8.33 \mathrm{r}\end{array}$ & $*$ & \\
\hline Annelida & $\begin{array}{l}\text { Hirudinea } \\
\text { Oligochaeta }\end{array}$ & $\begin{array}{c}1 \\
359\end{array}$ & $\begin{array}{c}8.33 \mathrm{r} \\
100.00 \mathrm{C}\end{array}$ & $\begin{array}{c}0 \\
169\end{array}$ & $\begin{array}{c}0.00 \\
91.67 \mathrm{C}\end{array}$ & & \\
\hline Arthropoda & Acarina & 9 & $25.00 \mathrm{c}$ & 2 & $8.33 r$ & & \\
\hline Crustácea & Gammaridae & 2 & $8.33 r$ & 0 & 0.00 & & \\
\hline Collembola & $\begin{array}{l}\text { Isotomidae } \\
\text { Hypogastruridae } \\
\text { Sminthuridae }\end{array}$ & $\begin{array}{c}58 \\
16 \\
2\end{array}$ & $\begin{array}{l}66.67 \mathrm{C} \\
25.00 \mathrm{c} \\
16.67 \mathrm{c}\end{array}$ & $\begin{array}{l}3 \\
1 \\
0\end{array}$ & $\begin{array}{c}25.00 \mathrm{c} \\
8.33 \mathrm{r} \\
0.00\end{array}$ & & \\
\hline Odonata & Libellulidae & 0 & 0.00 & 1 & $8.33 \mathrm{r}$ & & \\
\hline Ephemeroptera & $\begin{array}{l}\text { Baetidae } \\
\text { Leptophlebiidae } \\
\text { Leptohyphidae }\end{array}$ & $\begin{array}{c}129 \\
3 \\
597\end{array}$ & $\begin{array}{c}100.00 \mathrm{C} \\
8.33 \mathrm{r} \\
50.00 \mathrm{c}\end{array}$ & $\begin{array}{c}1921 \\
2 \\
232\end{array}$ & $\begin{array}{l}83.33 \mathrm{C} \\
16.67 \mathrm{c} \\
50.00 \mathrm{c}\end{array}$ & $*$ & $\begin{array}{l}* * \\
* *\end{array}$ \\
\hline Plecoptera & Perlidae & 5 & $16.67 \mathrm{c}$ & 0 & 0.00 & & \\
\hline Coleoptera & $\begin{array}{l}\text { Hydrophilidae } \\
\text { Dytiscidae } \\
\text { Staphylinidae } \\
\text { Elmidae } \\
\text { Curculionidae } \\
\text { Psephenidae } \\
\text { Chrysomelidae }\end{array}$ & $\begin{array}{c}10 \\
0 \\
8 \\
701 \\
4 \\
1 \\
1\end{array}$ & $\begin{array}{c}58.33 \mathrm{C} \\
0.00 \\
41.67 \mathrm{c} \\
83.33 \mathrm{C} \\
16.67 \mathrm{c} \\
8.33 \mathrm{r} \\
8.33 \mathrm{r}\end{array}$ & $\begin{array}{c}7 \\
34 \\
2 \\
293 \\
1 \\
0 \\
0\end{array}$ & $\begin{array}{c}8.33 \mathrm{r} \\
25.00 \mathrm{c} \\
16.67 \mathrm{c} \\
83.33 \mathrm{C} \\
8.33 \mathrm{r} \\
0.00 \\
0.00\end{array}$ & & \\
\hline Trichoptera & $\begin{array}{l}\text { Hydrobiosidae } \\
\text { Hydroptilidae } \\
\text { Limnephilidae } \\
\text { Polycentropodidae } \\
\text { Hydropsychidae }\end{array}$ & $\begin{array}{c}3 \\
5 \\
2 \\
3 \\
959\end{array}$ & $\begin{array}{c}16.67 \mathrm{c} \\
8.33 \mathrm{r} \\
8.33 \mathrm{r} \\
8.33 \mathrm{r} \\
33.33 \mathrm{c}\end{array}$ & $\begin{array}{c}2 \\
1 \\
1 \\
0 \\
100\end{array}$ & $\begin{array}{c}8.33 \mathrm{r} \\
8.33 \mathrm{r} \\
8.33 \mathrm{r} \\
0.00 \\
41.67 \mathrm{c}\end{array}$ & & \\
\hline Diptera & $\begin{array}{l}\text { Blephariceridae } \\
\text { Ceratopogonidae } \\
\text { Chironomidae } \\
\text { Psychodidae } \\
\text { Simuliidae } \\
\text { Dolichopodidae } \\
\text { Empididae } \\
\text { Muscidae } \\
\text { Tabanidae } \\
\text { Ephydridae } \\
\text { Sciomyzidae } \\
\text { Tipulidae }\end{array}$ & $\begin{array}{c}25 \\
5 \\
1074 \\
21 \\
64 \\
1 \\
20 \\
0 \\
2 \\
8 \\
5 \\
5\end{array}$ & $\begin{array}{c}25.00 \mathrm{c} \\
25.00 \mathrm{c} \\
100.00 \mathrm{C} \\
41.67 \mathrm{c} \\
75.00 \mathrm{C} \\
8.33 \mathrm{r} \\
58.33 \mathrm{C} \\
0.00 \\
8.33 \mathrm{r} \\
41.67 \mathrm{c} \\
25.00 \mathrm{c} \\
5.00 \mathrm{r}\end{array}$ & $\begin{array}{c}85 \\
4 \\
16335 \\
108 \\
20 \\
4 \\
168 \\
2 \\
0 \\
6 \\
1 \\
0\end{array}$ & $\begin{array}{c}66.67 \mathrm{C} \\
16.67 \mathrm{c} \\
100.00 \mathrm{C} \\
75.00 \mathrm{C} \\
58.33 \mathrm{C} \\
16.67 \mathrm{c} \\
100.00 \mathrm{C} \\
16.67 \mathrm{c} \\
0.00 \\
25.00 \mathrm{c} \\
8.33 \mathrm{r} \\
0.00\end{array}$ & $*$ & \\
\hline
\end{tabular}

$C=$ Constante $>50 \%$ de ocurrencia; $c=$ común, entre 10 y $50 \%$ de ocurrencia; $r=$ rara, $<10 \%$ de ocurrencia

$*=$ valores significativos entre promedios de época de avenida y estiaje; $* *=$ valores significativos entre promedios menores a 1000 msnm y mayores de 1000 msnm 
Cuadro 4. Parámetros comunitarios y de calidad de agua de los macroinvertebrados bentónicos (MIB) en época de avenida y estiaje de la cuenca del río Rímac, Lima-Perú, 2009

\begin{tabular}{|c|c|c|c|c|c|c|c|c|c|c|c|c|c|c|c|c|c|c|}
\hline \multirow[b]{2}{*}{ 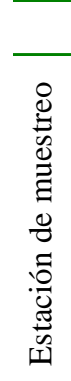 } & \multicolumn{10}{|c|}{ Avenida - 2009} & \multicolumn{8}{|c|}{ Estiaje - 2009} \\
\hline & 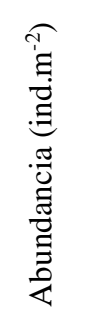 & & 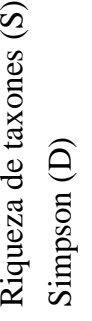 & 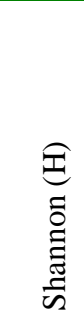 & $\begin{array}{l}\hat{\theta} \\
\frac{0}{0} \\
\frac{0}{2}\end{array}$ & $\stackrel{5}{\stackrel{5}{1}}$ & 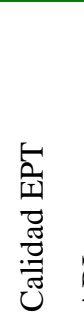 & $\bar{q}$ & 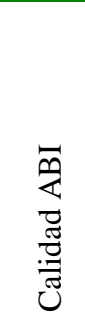 & 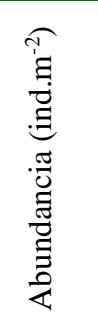 & 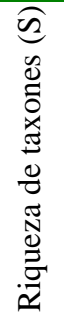 & 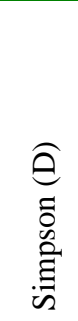 & 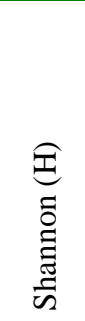 & $\begin{array}{l}E \\
\frac{\partial}{0} \\
\frac{0}{2}\end{array}$ & $\stackrel{5}{5}$ & 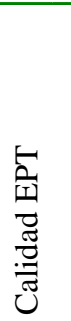 & $\bar{q}$ & 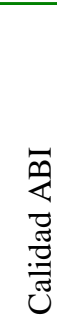 \\
\hline$\overline{\mathrm{E} 1}$ & 552 & 10 & 0.48 & 1.08 & 0.47 & 7.40 & $\mathrm{M}$ & 34 & Mod & 1659 & 7 & 0.95 & 0.16 & 0.08 & 0.00 & $M$ & 16 & $\mathrm{M}$ \\
\hline $\mathrm{E} 2$ & 3733 & 22 & 0.53 & 0.99 & 0.32 & 2.50 & M & 70 & MB & 5989 & 16 & 0.90 & 0.30 & 0.11 & 0.60 & M & 46 & B \\
\hline E3 & 570 & 8 & 0.39 & 1.21 & 0.58 & 0.70 & M & 12 & M & 2500 & 5 & 0.79 & 0.49 & 0.30 & 0.00 & M & 13 & M \\
\hline $\mathrm{E} 4$ & 159 & 6 & 0.32 & 1.33 & 0.74 & 4.70 & M & 10 & $\mathrm{P}$ & 2930 & 9 & 0.84 & 0.42 & 0.19 & 2.90 & $\mathrm{M}$ & 26 & M \\
\hline E5 & 459 & 11 & 0.47 & 1.30 & 0.54 & 13.70 & $\mathrm{M}$ & 43 & Mod & 10867 & 12 & 0.86 & 0.36 & 0.15 & 4.50 & M & 46 & B \\
\hline E6 & 404 & 17 & 0.23 & 1.99 & 0.70 & 11.00 & $\mathrm{M}$ & 57 & B & 5748 & 6 & 0.79 & 0.47 & 0.26 & 0.10 & M & 24 & M \\
\hline E7 & 241 & 17 & 0.14 & 2.35 & 0.83 & 23.10 & $M$ & 53 & B & 8530 & 12 & 0.68 & 0.74 & 0.30 & 11.10 & $\mathrm{M}$ & 56 & B \\
\hline E8 & 248 & 12 & 0.20 & 1.94 & 0.78 & 20.90 & $M$ & 43 & Mod & 6578 & 10 & 0.56 & 0.87 & 0.38 & 24.80 & $\mathrm{M}$ & 46 & B \\
\hline E9 & 259 & 13 & 0.16 & 2.09 & 0.81 & 13.90 & $M$ & 41 & Mod & 9878 & 10 & 0.70 & 0.64 & 0.28 & 13.20 & M & 46 & B \\
\hline E10 & 6556 & 13 & 0.36 & 1.27 & 0.49 & 69.60 & $B$ & 55 & B & 7544 & 12 & 0.49 & 0.88 & 0.35 & 37.40 & $0 \mathrm{R}$ & 53 & B \\
\hline E11 & 1900 & 17 & 0.29 & 1.62 & 0.57 & 53.60 & $B$ & 66 & B & 8163 & 13 & 0.75 & 0.60 & 0.23 & 11.10 & $0 \mathrm{M}$ & 57 & B \\
\hline E12 & 448 & 15 & 0.40 & 1.48 & 0.54 & 75.40 & MB & 56 & B & 1896 & 11 & 0.77 & 0.57 & 0.24 & 8.00 & $\mathrm{M}$ & 46 & B \\
\hline
\end{tabular}

$(<1000 \mathrm{msnm}=19.62 \pm 0.67 ;>1000 \mathrm{msnm}=$ $13.75 \pm 1.26, \mathrm{t}=4.08 ; \mathrm{p}<0.001), \mathrm{EPT}(<1000$ $\mathrm{msnm}=30.17 \pm 6.81 ;>1000 \mathrm{msnm}=$ $4.00 \pm 1.31, \mathrm{t}=3.76 ; \mathrm{p}=0.003)$ y $\mathrm{ABI}(<1000$ $\mathrm{msnm}=51.50 \pm 2.07 ;>1000 \mathrm{msnm}=$ $33.08 \pm 5.62, \mathrm{t}=3.07 ; \mathrm{p}=0.008)$. Se observó que el 75 y $91.7 \%$ de las estaciones presentaron un EPT de calidad mala en época de avenida y de estiaje, respectivamente. En cambio, para ABI se clasificó de calidad pésima-mala en época de avenida (16.7\%) y de estiaje (33.3\%), respectivamente.

Según el índice de Morisita-Horn, en la época de avenida se asocian las estaciones de muestreo en tres grupos: $\mathrm{A}\left(\mathrm{E}_{1} \mathrm{a}_{6}\right), \mathrm{B}$ $\left(\mathrm{E}_{7}\right.$ a $\left.\mathrm{E}_{9}\right)$ y $\mathrm{C}\left(\mathrm{E}_{10}\right.$ a $\left.\mathrm{E}_{12}\right)$, mientras que para la temporada de estiaje hay mayor similaridad y solo dos grupos, restringiéndose el rango, donde se muestra que las estaciones son en más del >90\% de los casos (Figura 2).

\section{Ensayos Ecotoxicológicos}

En los ensayos toxicológicos de las aguas con D. magna se encontraron valores de $\mathrm{CL}_{50}$ menores al $100 \%$ a $48 \mathrm{~h}$ solo en $\mathrm{E}_{1} \mathrm{y}$ LOEC de $6.25 \%$ en nueve estaciones en la época de avenida. Por otro lado, para la época de estiaje ninguna estación señaló valores de $\mathrm{CL}_{50}$ menores al $100 \%$ a $48 \mathrm{~h}$ y solo las estaciones $\mathrm{E}_{9}$ y $\mathrm{E}_{12}$ presentaron LOEC significativos (Cuadro 5).

Para los ensayos toxicológicos de sedimento con $C$. calligraphus se encontró $\mathrm{CL}_{50}$ de mortalidad, LOEC de mortalidad y $\mathrm{CE}_{50}$ de disminución de la biomasa en la época de avenida a los 10 días de exposición en la estación de muestreo $\mathrm{E}_{1}$ (Cuadro 5). Los valores de LOEC mostraron efecto en cuatro estaciones de E durante la época de avenida. 
Cuadro 5. Valores de LOEC y $\mathrm{CL}_{50}(\%)$ a las $48 \mathrm{~h}$ de exposición obtenidos de los ensayos ecotoxicológicos de mortalidad con Daphnia magna con las aguas del río Rímac, y de mortalidad $(\%)$ y biomasa $(\mu \mathrm{g})$ con Chironomus calligraphus con sedimento del río Rímac en avenida y estiaje, Lima, Perú (2009)

\begin{tabular}{|c|c|c|c|c|c|c|c|c|c|c|c|c|c|}
\hline Época & Parámetro & $\mathrm{E}_{1}$ & $\mathrm{E}_{2}$ & $\mathrm{E}_{3}$ & $\mathrm{E}_{4}$ & $\mathrm{E}_{5}$ & $\mathrm{E}_{6}$ & $\mathrm{E}_{7}$ & $\mathrm{E}_{8}$ & $\mathrm{E}_{9}$ & $\mathrm{E}_{10}$ & $\mathrm{E}_{11}$ & $\mathrm{E}_{12}$ \\
\hline \multirow[t]{2}{*}{ Avenida 09} & $\begin{array}{l}\text { LOEC }(\%) \mathrm{de} \\
\text { D. magna }\end{array}$ & 6.25 & 6.25 & 6.25 & 6.25 & 6.25 & * & 6.25 & $*$ & 6.25 & * & 6.25 & 6.25 \\
\hline & $\begin{array}{l}\mathrm{CL}_{50} \text { de } D . \\
\text { magna }\end{array}$ & 17.10 & $*$ & $*$ & $*$ & $*$ & $*$ & $*$ & $*$ & $*$ & $*$ & $*$ & $*$ \\
\hline \multirow[t]{2}{*}{ Estiaje 09} & $\begin{array}{l}\text { LOEC de } D . \\
\text { magna }\end{array}$ & $*$ & $*$ & $*$ & $*$ & $*$ & $*$ & $*$ & $*$ & 25 & $*$ & $*$ & 6.25 \\
\hline & $\begin{array}{l}\mathrm{CL}_{50} \text { de } D . \\
\text { magna }\end{array}$ & $*$ & $*$ & $*$ & $*$ & $*$ & $*$ & $*$ & $*$ & $*$ & $*$ & $*$ & $*$ \\
\hline \multirow[t]{3}{*}{ Avenida 09} & $\begin{array}{l}\text { CL }_{50} \\
\text { mortalidad } \\
\text { 10d de } C \text {. } \\
\text { calligraphus }\end{array}$ & 70.70 & $*$ & $*$ & $*$ & $*$ & $*$ & $*$ & $*$ & $*$ & $*$ & $*$ & $*$ \\
\hline & $\begin{array}{l}\text { LOEC } \\
\text { mortalidad } \\
\text { 10d de } C . \\
\text { calligraphus }\end{array}$ & 6.25 & $*$ & $*$ & $*$ & $*$ & 6.25 & 50 & $*$ & $*$ & $*$ & $*$ & 50 \\
\hline & $\begin{array}{l}\mathrm{CE}_{50} \text { biomasa } \\
\text { 10d de } C . \\
\text { calligraphus }\end{array}$ & 70.71 & $*$ & $*$ & $*$ & $*$ & $*$ & $*$ & $*$ & $*$ & * & $*$ & $*$ \\
\hline \multirow[t]{3}{*}{ Estiaje 09} & $\begin{array}{l}\text { CL50 }_{50} \\
\text { mortalidad } \\
\text { 10d de } C \text {. } \\
\text { calligraphus }\end{array}$ & $*$ & $*$ & $*$ & $*$ & $*$ & * & $*$ & $*$ & $*$ & $*$ & $*$ & $*$ \\
\hline & $\begin{array}{l}\text { LOEC } \\
\text { mortalidad } \\
10 \mathrm{~d}(\%)\end{array}$ & $*$ & $*$ & $*$ & $*$ & $*$ & $*$ & $*$ & $*$ & $*$ & $*$ & $*$ & $*$ \\
\hline & $\begin{array}{l}\mathrm{CE}_{50} \text { biomasa } \\
\text { 10d de } C . \\
\text { calligraphus }\end{array}$ & $*$ & $*$ & $*$ & $*$ & $*$ & $*$ & $*$ & $*$ & $*$ & $*$ & $*$ & $*$ \\
\hline
\end{tabular}

* Valor de toxicidad (LOEC, $\mathrm{CL}_{50}$ o $\mathrm{CE}_{50}$ ) mayor que $100 \%$, por lo que no fue determinado

En la época de estiaje no mostró ningún efecto en la $\mathrm{CL}_{50}$ de mortalidad, LOEC de mortalidad y $\mathrm{CE}_{50}$ de disminución de la biomasa en los sedimentos (Cuadro 5).

\section{Análisis Integral}

El análisis de correspondencia canónica (ACC) (Figura 3) indica la relación entre- las variables ambientales y los índices comunitarios de calidad de agua de los MIB y ecotoxicológicos de la cuenca del río Rímac. El pH y el caudal se asocian más con el ABI, $\mathrm{D}, \mathrm{S}, \mathrm{H}, \mathrm{J}$ y $D$. magna; en cambio, $C$. calligraphus y EPT no se asocian con las variables físicas y químicas. El OD y la transparencia se relacionan con la abundancia de MIB. 


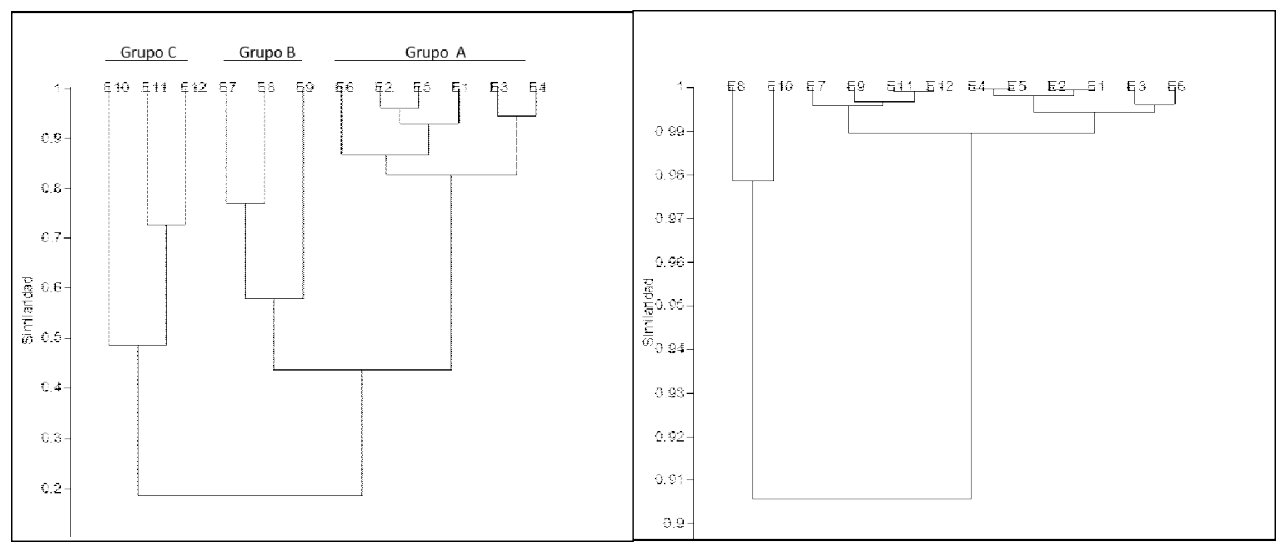

Figura 2. Dendrograma de similaridad con el índice de Morisita-Horn empleando el programa estadístico Past, en base a los macroinvertebrados bentónicos (MIB) colectados en la época de avenida (figura izquierda) y época de estiaje (figura derecha) del río Rímac, Lima-Perú (2009)

\section{Discusión}

\section{Parámetros Fisicoquímicos}

La época de avenida presentó mayores valores de $\mathrm{pH}$ y caudal y, en cambio, mayores valores de CE, ST, OD y transparencia en la época de estiaje. En el caso del OD en los ecosistemas lóticos, su valor depende de factores ambientales como la temperatura, altitud, características del caudal (flujo de turbulencia) y presión atmosférica (GuerreroBolaño et al., 2003). Valores bajos de $\mathrm{pH}$ pudieran tener efectos negativos en los organismos acuáticos e indicar posible contaminación del cuerpo de agua (Guerrero-Bolaño et al., 2003). Sin embargo, el $\mathrm{pH}$ puede estar influenciado por el tipo de suelo y vegetación circundante a un cuerpo de agua. En el estudio, el $\mathrm{pH}$ más bajo fue registrado para la estación 8 , la cual se dice que es atóxica, en tanto que la $\mathrm{E}_{1}$ presentó un $\mathrm{pH}$ de 7.7 en la época seca. Righi-Cavallaro et al. (2010) encontraron estacionalidad de la $\mathrm{CE}$, pero el $\mathrm{pH}$ y la temperatura no mostraron ningún patrón relacionado con la avenida-estiaje en un cuerpo de agua lótico neotropical en Brasil. El aumento del $\mathrm{pH}$ en la época de avenida se asocia con el incremento de $\mathrm{S}, \mathrm{H}^{\prime}$ y $\mathrm{J}^{\prime}$, y con una disminución de la abundancia y D en el mismo periodo.

Solo se observaron diferencias de temperatura con la variación altitudinal. En este sentido, Guerrero-Bolaño et al. (2003) mostraron que la temperatura en los trópicos presenta variaciones en su valor en dependencia con la altitud.

\section{Macroinvertebrados Bentónicos (MIB)}

Diferencias en la abundancia entre épocas de avenida y estiaje solo se observaron para Planorbidae, Baetidae, Chironomidae, Empididae y Tipulidae. Estas cinco familias presentan según la escala de tolerancia de ABI valores bajos entre 2 y 5 puntos, por lo que serían taxones considerados con una amplia capacidad de colonización, pero influenciados por la estacionalidad de la precipitación y el caudal del cuerpo de agua. Así, en el sentido estricto no serían indicadores ideales de sensibilidad a la contaminación, pero 


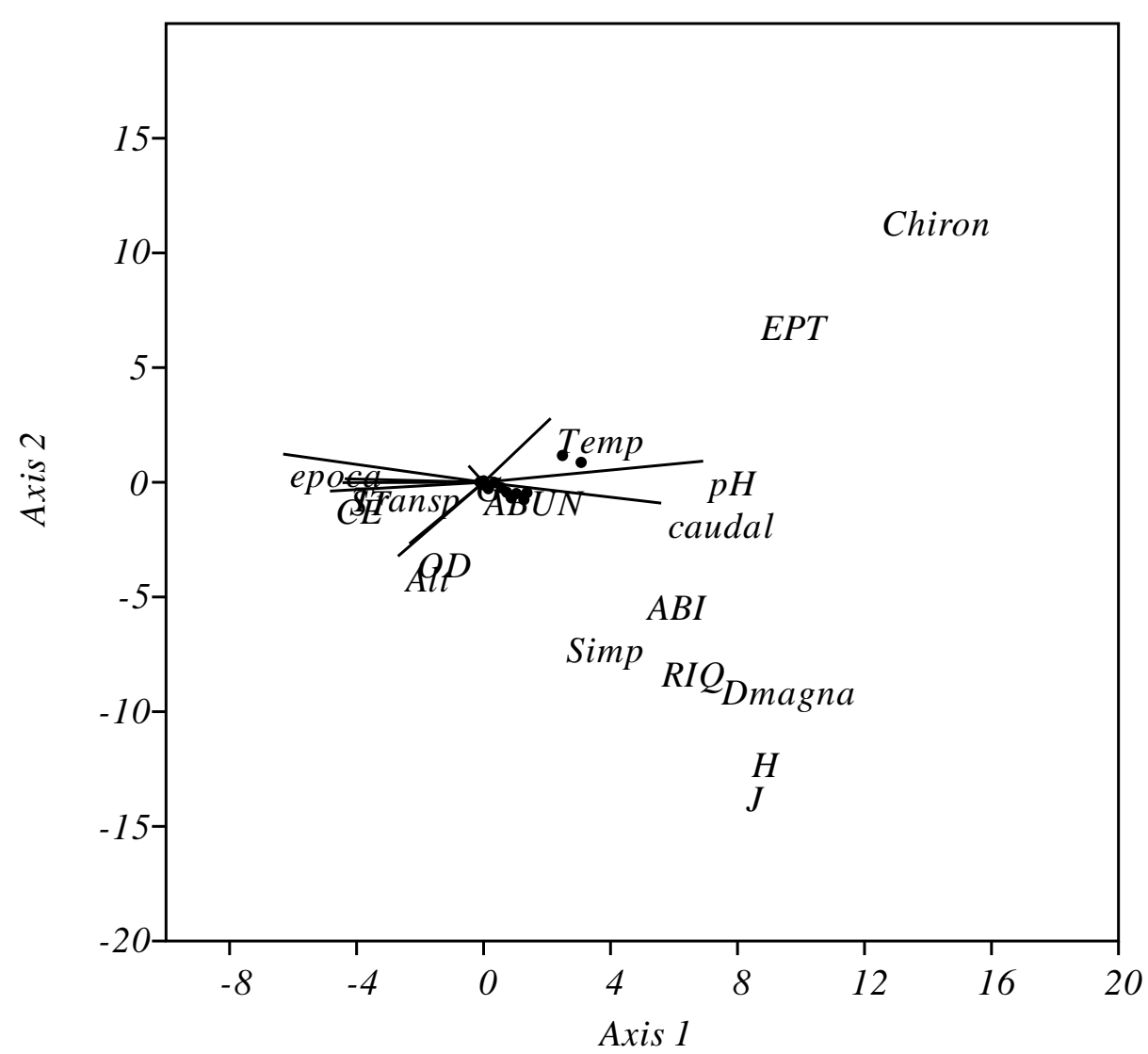

Figura 3. Análisis de Correspondencia Canónica (ACC) para la relación entre las variables ambientales y los índices comunitarios de calidad de agua de los macroinvertebrados bentónicos (MIB) y ecotoxicológicos en la cuenca del río Rímac, Lima, Perú (2009). Chiro = Chironomus calligraphus $; \mathrm{J}=$ Equidad de Pielou; $\mathrm{H}^{\prime}=$ Shannon-Wiener; RIQ = Riqueza $(\mathrm{S}) \mathrm{M}$ Simp = Dominancia de Simpson (D); Dmagna = Daphnia magna $;$ Alt $=$ Altitud $; \mathrm{ABUN}=$ Abundancia $\left(\right.$ ind $\left.\cdot \mathrm{m}^{2}\right) ;$ Temp $=$ Temperatura $;$ Transp $=$ Transparencia $; \mathrm{CE}=$ Conductividad eléctrica $; \mathrm{EPT}=$ Ephemeroptera/Plecoptera/ Trichoptera

si a los periodos estacionales de avenida y estiaje (Guerrero-Bolaño et al., 2003; Trama et al., 2009; Alomía et al., 2017).

El aumento de Planorbidae y Tipulidae en época de avenida podría explicarse por las condiciones del sistema acuático neotropical. Planorbidae es una familia de moluscos considerada de mayor importancia en la vegetación marginal del río Rímac y que podría incrementar en el periodo lluvioso, como es señalado para varias especies de
Planorbidos en ríos de México (CózatlManzano y Naranjo-García, 2007). Sin embargo, varios autores señalan que los moluscos acuáticos son altamente sensibles a la remoción por inundaciones en el periodo lluvioso, pero altamente tolerantes a bajas concentraciones de OD (Righi-Cavallaro et al., 2010, Souto et al., 2011). Righi-Cavallaro et al. (2010) señalan que las familias Tipulidae, Chironomidae, Empididae son taxones indicadores para etapas de estiaje en ambientes lóticos neotropicales. 
Las familias Baetidae, Chironomidae y Empididae aumentaron en la época de estiaje. Gonçalves y de Menezes (2011) encontraron en varias evaluaciones en ríos de Brasil patrones opuestos en las abundancias de Baetidae y Chironomidae. Baetidae es considerada una familia con un amplio rango de tolerancia a perturbaciones del hábitat (Guerrero-Bolaño et al., 2003). La dominancia de Baetidae en la época de estiaje podría estar relacionada a la presencia de vegetación y a una mayor disponibilidad de sustratos duros (piedras) en las estaciones que servirían de sustrato, debido a que carece de adaptaciones morfológicas para altos caudales como en las épocas de avenida; además de un ciclo de desarrollo bastante rápido (Gonçalves y de Menezes, 2011; Ríos-Touma et al., 2011). La familia Chironomidae fue más abundante en la temporada de estiaje. Según Alonso y Camargo (2005), la abundancia de individuos de esta familia está relacionado a la contaminación por materia orgánica, debido a que son tolerantes a este tipo de contaminación. Así mismo, según Roldán (1992), el aumento de Chironomidae y otros dípteros incrementan por influencia del control antropogénico del flujo de los ríos, por la descarga de contaminantes industriales y domésticos (Pave y Marchese, 2005; Oscoz et al., 2006), así como por las actividades ganaderas en los alrededores del río que contribuyen a la degradación de los ecosistemas acuáticos. Trama et al. (2009) indican que los Chironomidos son uno de los grupos más dominantes y tolerantes a condiciones anóxicas debido a la presencia de hemoglobina, lo cual le permite dominar el bentos en condiciones adversas (Iannacone et al., 2003; Ríos-Touma et al., 2011). Opuestamente, Oscoz et al. (2006) encontraron una disminución del caudal en un río en España y una disminución en la abundancia de MIB.

La Abundancia y D fueron mayores en la temporada de estiaje, mientras que $\mathrm{S}, \mathrm{H}^{\prime} \mathrm{y}$ $\mathrm{J}^{\prime}$ fueron mayores en la temporada de avenida. Ríos-Touma et al. (2011) encontraron que la composición (riqueza y diversidad) de MIB en Ecuador estuvo asociada al régimen hidrológico incrementándose en la época seca, debido a la mayor estabilidad de este régimen. Gonçalves y de Menezes (2011) no observaron relación entre la abundancia de MIB y las precipitaciones en río de Brasil. Los MIB que están asociados a la disponibilidad de alimento presente en el medio acuático son capaces de reflejar variaciones a las perturbaciones antropogénicas e hidrológicas naturales (Pave y Marchese, 2005; Righi-Cavallaro et al., 2010; Rios-Touma et al., 2011).

El incremento de materia orgánica en el agua produce una proliferación de los microorganismos encargados de su descomposición, lo que genera entre otros efectos una reducción de la concentración de oxígeno disuelto en el agua y un aumento de la concentración de nutrientes inorgánicos, como el amonio y el fosfato (Alonso y Camargo, 2005). Esto explicaría la diferencia de la abundancia de la comunidad de los MIB entre la época de venida y estiaje, siendo mayor en la época de estiaje en todas las estaciones de muestreo. El alimento potencial y el sustrato para los MIB están más disponibles en la época seca, al igual que una menor disponibilidad de hábitat $\mathrm{y}$, como consecuencia, un aumento en la agregación de los individuos (Righi-Cavallaro et al., 2010) y en este estudio, la agregación de materia orgánica proveniente de actividades antropogénicas fue un fenómeno recurrente. Opuestamente, Trama et al. (2009) encontró una mayor riqueza de invertebrados en la época de estiaje en el humedal Palo Verde, Costa Rica, atribuyéndolo a la hidrología y a los cambios ambientales del sistema, pero en cambio, en forma similar a este estudio, encontraron una mayor abundancia de invertebrados y de OD en la época de estiaje. Guerrero-Bolano et al. (2003) indicaron perturbación de la biota de MIB con relación a la estacionalidad lluviosa por remoción y falta de estabilidad del sustrato en el cuerpo de agua (Righi-Cavallaro et al., 2010), lo que estaría ocurriendo frecuentemente en el río Rímac. 
Se observó que el 75 y $91.7 \%$ de las E presentaron un EPT de calidad mala en época de avenida y de estiaje, respectivamente. En cambio, para ABI se clasificó de calidad pésima-mala en época de avenida (16.67\%) y de estiaje (33.33\%), respectivamente. Figueroa et al. (2007) señalan que en los periodos de bajo caudal en los sistemas fluviales se expresa mejor la variación y deterioro de la calidad de las aguas. El presente estudio muestra, según los índices EPT y ABI, que las aguas del río Rímac en periodo de estiaje se encuentran deterioradas, expresándose con la baja $\mathrm{H}^{\prime}$ y $\mathrm{J}$, alta $\mathrm{D}$ de algunos grupos y disminución de la $\mathrm{S}$. Por su lado, Gonçalves y de Menezes (2011) argumentan que los índices bióticos con MIB decrecen con el incremento del caudal en periodos lluviosos.

Estas diferencias no pueden ser atribuidas a los métodos de muestreo que fueron similares para la época de avenida y estiaje. Los índices bióticos empleados (EPT y ABI) valoran de mejor calidad a las aguas del río Rímac en la temporada de avenida. Según Rosemberg y Resh (1993), el índice EPT es adecuado como un índice preliminar para valorar la perturbación por minería; no obstante, no existe un índice específico de biovaloración basada en esta comunidad. Gonçalves y de Menezes (2011) señalan que el EPT es más sensible a la contaminación orgánica. El ABI se adecúa a las comunidades de la cuenca estudiada, ya que este índice incluye a la mayoría de los taxones encontrados durante en estudio. MuñozReveaux et al. (2003) señalan que los índices bióticos de calidad de agua en base a MIB pueden sufrir variaciones estacionales relacionadas a los periodos de seca-lluviosos. En el presente estudio, los índices EPT y ABI en el río Rímac no presentaron diferencias significativas relacionadas a la estacionalidad (periodo de avenida y estiaje).

Algunos autores señalan que los índices bióticos en base a MIB varían de forma similar a la $\mathrm{S}, \mathrm{H}^{\prime}$ y $\mathrm{D}$, y pudieran tener bue- nas correlaciones en dependencia con la estacionalidad (Muñoz-Reveaux et al., 2003). En la presente investigación se observó según el $\mathrm{ACC}$ que el $\mathrm{ABI}, \mathrm{D}, \mathrm{S}, \mathrm{H}^{\prime}$ y $\mathrm{J}^{\prime}$ se encontraron asociados (Figura 3). En especial, el índice biótico de calidad de agua ABI es una buena herramienta para la evaluación de la calidad del agua en los ambientes lóticos en el Perú, al ahorrar tiempo y recursos económicos debido a su simplicidad y baja resolución taxonómica (Figueroa et al., 2003; Muñoz-Reveaux et al., 2003; Oscoz et al., 2006). De igual forma se espera una adecuada correlación con algunas variables fisicoquímicas (estresores antropogénicos), pero en este trabajo solo el ABI observó una buena asociación con el $\mathrm{pH}$ y el caudal (Figura 3). Figueroa et al. (2003) encontraron correlaciones entre la $\mathrm{CE}, \mathrm{OD}, \mathrm{DBO}, \mathrm{T}^{\circ} \mathrm{C}$ y el índice biótico de calidad de agua en base a MIB; sin embargo, para una adecuada interpretación del $\mathrm{ABI}$ se sugiere complementar con caracterizaciones de la columna de agua, peces y actividades productivas desarrolladas en la cuenca debido a que al estar solo basado en presencia-ausencia no considera las abundancias de cada taxón y llegar así a conclusiones fidedignas (Figueroa et al., 2003; Guerrero-Bolaño et al., 2003).

En el dendrograma de similaridad realizado con el índice de Morisita-Horn se aprecia la formación de tres grupos en la época de avenida, los cuales podrían indicar: grupo A respuesta a los pasivos mineros, grupo B respuesta a impacto doméstico y agricultura, y grupo $\mathrm{C}$ respuesta a impacto por la agricultura, industrias y doméstico (Iannacone et al., 2005; Bedregal et al., 2010; Llontop y Cabrera, 2010; Alomía et al., 2017), lo cual estaría relacionado a las actividades que se realizan en los sectores de la cuenca.

El área del río Rímac se diferencia en tres secciones: la primera es la zona de Bellavista (Huarochirí) que presenta un mayor impacto por la minería, lo cual es corroborado por Bedregal et al. (2010) quienes señalan que la cuenca alta de río Rímac (so- 
bre $3500 \mathrm{msnm}$ ) que incluye las tres primeras estaciones $\left(E_{1}\right.$ a $\left.E_{3}\right)$ presenta una intensa actividad minera con explotaciones de cobre, plomo, zinc, antimonio, oro y plata, con un volumen significativo de vertimientos descargados directamente al río, y la cuenca media (entre 1000 y $3500 \mathrm{msnm}$ ) donde el agua se usa para la agricultura y para centrales hidroeléctricas $\left(\mathrm{E}_{4}\right.$ a $\left.\mathrm{E}_{6}\right)$. La segunda sección $\left(\mathrm{E}_{7}\right.$ a $\left.\mathrm{E}_{9}\right)$ desde Matucana hasta Huachipa tiene un mayor impacto por la agricultura y desechos domésticos, donde Bedregal et al. (2010) indican que forma parte de la cuenca baja del río Rímac con actividad agrícola y el asentamiento de la ciudad de Lima.

Finalmente, desde Naña hasta la bocatoma de agua de la Atarjea $\left(\mathrm{E}_{10}\right.$ a $\left.\mathrm{E}_{12}\right)$ se observa el mayor impacto por industrias, asentamientos humanos y poblados situados en las riberas de río, que se caracterizan por la ausencia de sistemas de tratamiento de desagües y alcantarillado y, por lo tanto, son descargadas directamente al río. Bedregal et al. (2010) señalan que en esta área existen fábricas de productos químicos, textiles, papeleras, alimento, curtiembres, cerveza, etc. Sin embargo, en la época de estiaje, todas las estaciones de muestreo muestran un alto grado de similaridad (>97\%), lo que estaría indicando que la seria contaminación a lo largo del río Rímac se hace homogénea. Asimismo, se considera que el caudal ecológico estaría jugando un rol importante en la dinámica biológica del rio Rímac. Pan et al. (2015) consideran que la credibilidad de los resultados de los caudales ecológicos es influenciada por la falta de una base ecológica en los análisis hidráulicos e hidrológicos, y por los requerimientos de agua en el ecosistema de un río con un adecuado enfoque global.

\section{Ensayos Ecotoxicológicos}

En cuanto a los ensayos ecotoxicológicos de las aguas con D. magna, se encontró en la época de avenida valores de $\mathrm{CL}_{50}$ menores al $100 \%$ a $48 \mathrm{~h}$ solo en $\mathrm{E}_{1}$, y LOEC de
$6.25 \%$ en nueve estaciones de muestreo. Para la época de estiaje ninguna estación señaló valores de $\mathrm{CL}_{50}$ menores al $100 \%$ a $48 \mathrm{~h}$ y solo las estaciones $\mathrm{E}_{9} \mathrm{y}_{12}$ presentaron LOEC significativos. De Schamphelaere y Janssen (2002) indican que altas concentraciones de $\mathrm{Ca}$ y $\mathrm{Mg}$ en los ecosistemas acuáticos protegen de la toxicidad de metales, en tanto que Jin-Yong et al. (2017) reportaron mayores efectos protectores del $\mathrm{Ca}$ en comparación al $\mathrm{Mg}$, al evaluar la toxicidad del cobre en Daphnia galeata Sars, 1864. Asimismo, Álvarez-Manzaneda et al. (2017) registraron un efecto en la reproducción partenogenética de D. magna en presencia de Fe disuelto. En este estudio no se ha contemplado la evaluación de la dureza del agua, por lo que futuras investigaciones deberán contemplar este parámetro.

Para la evaluación de riesgos ambientales se ha empleado las escalas ecotoxicológicas de Munkittrick et al. (1991) y Arambasic et al. (1995). Para el caso de aguas, según Munkittrick, la estación de muestreo $\mathrm{E}_{1}\left(\mathrm{CL}_{50-48 \mathrm{~h}}=17.01 \%\right)$ es clasificada como altamente tóxico y según Arambasic como politóxica. Este resultado podría explicarse a la alta actividad minera realizada en esta estación. Llontop y Cabrera (2010) señalan que el área de estudio donde se localiza $E_{1}$ es una zona con actividad minera polimetálica y con pasivos ambientales mineros entre bocaminas y relaveras. Durante 2008 se registró en la época de avenida en esta $\mathrm{E}$, valores de metales pesados sobre la normatividad vigente de $\mathrm{Cd}, \mathrm{Pb}$ y Mn. MINSA (2010) registró en la época de estiaje (agosto-2009) valores altos de Al, Mn, $\mathrm{Fe}$ y $\mathrm{Pb}$ en las muestras de agua en la estación $\mathrm{E}_{1}$. Sin embargo, en este estudio no se observaron valores significativos de $\mathrm{CL}_{50}$ en la época de estiaje para la muestra de agua evaluada con D. magna en $\mathrm{E}_{1}$. Las estaciones $\mathrm{E}_{9} \mathrm{y}_{12}$ presentaron LOEC significativos para la muestra de agua evaluada con $D$. magna durante el estiaje. La mayoría de las estaciones son clasificadas como atóxicas en 
base a la $\mathrm{CL}_{50}$ en ambas escalas toxicológicas. Se puede observar que las aguas de avenida de la $\mathrm{E}_{1}$ fue la más afectada de la perspectiva toxicológica. Este punto presentó además altos valores de CE y temperaturas bajas $\left(<10^{\circ} \mathrm{C}\right)$, ocasionando bajos valores de $\mathrm{J}^{\prime}, \mathrm{S}$ y abundancia de MIB, EPT calificada como mala y el ABI como moderado.

Para los ensayos ecotoxicológicos de sedimento con $C$. calligraphus se encontró $\mathrm{CL}_{50}$ de mortalidad, LOEC de mortalidad y $\mathrm{CE}_{50}$ de disminución de la biomasa en la época de avenida a los 10 días de exposición en la estación de muestreo $\mathrm{E}_{1}$. Los valores de LOEC mostraron efecto en cuatro estaciones de muestreo durante la época de avenida. En la época de estiaje no mostró ningún efecto en la $\mathrm{CL}_{50}$ de mortalidad, LOEC de mortalidad y $\mathrm{CE}_{50}$ de disminución de la biomasa en los sedimentos. ÁlvarezManzaneda et al. (2017) registraron que en presencia de Fe disuelto, no hubo mortalidad significativa de las larvas de Chironomus sp. Los resultados obtenidos en este estudio, según Munkittrick et al. (1991), la $\mathrm{E}_{1}$ es ligeramente tóxica y según Arambasic et al. (1991) como oligotóxica. Las demás estaciones son clasificadas como atóxicas en ambas escalas toxicológicas.

La toxicidad registrada en $\mathrm{E}_{1}$ para los sedimentos en la época de avenida, podría de igual forma deberse a la gran actividad minera histórica que se desarrolla en esta zona (Iannacone et al., 2005). Guerrero (2014) indica que esta estación presenta valores mayores a los estándares de calidad ambiental según normatividad holandesa para $\mathrm{Pb}$ y Zn. Rivera et al. (2007) indican que los sedimentos del río Rímac están contaminados por $\mathrm{As}, \mathrm{Cu}, \mathrm{Hg}, \mathrm{Zn}$ y $\mathrm{Sb}$ con valores sobre la normatividad holandesa; sin embargo, indican que el curso medio e inferior del río Rímac concentra la mayoría de los metales. La posible ausencia o baja toxicidad en las estaciones de muestreo restantes en base a la $\mathrm{CL}_{50}$ de $D$. magna y de $C$. calligraphus podría deberse a la capacidad de arrastre y al movimiento de las aguas de los sistemas lóticos, efectos que estarían amortiguando el grado de toxicidad que se observa en las estaciones de la parte alta del río. Iannacone et al. (2000) sugieren emplear larvas I de $C$. calligraphus para evaluar la calidad de aguas del río Rímac como una herramienta sensible para la evaluación de los sistemas acuáticos epicontinentales. Iannacone et al. (2005) evaluaron con C. calligraphus a los sedimentos elutriados del río Aruri y del lago Junín, Perú. En este estudio se sugiere el uso del estadio II de C. calligraphus para la evaluación de los sedimentos dulceacuícolas. Además, tiene como ventaja principal el ser un material biológico abundante y de permanente disponibilidad para la obtención de los primeros estadios larvales (Iannacone y Dale, 1999) y de fácil manipulación en este tipo de ensayo al conocerse su ciclo de vida en condiciones de laboratorio (Zilli et al., 2008; Canteiro y Albertoni, 2011).

En conclusión, los resultados muestran que el río Rímac, en el tramo evaluado, se encuentra seriamente contaminado. La época de avenida presentó mayores valores de $\mathrm{pH}$ y caudal. En cambio, mayores valores de CE, ST, OD y transparencia se observaron en la época de estiaje. Se observaron diferencias altitudinales con la temperatura. Diferencias en la abundancia entre épocas de avenida y estiaje solo se observaron para Planorbidae, Baetidae, Chironomidae, Empididae y Tipulidae. Los índices de diversidad y equitabilidad fueron mayores en época de avenida, pero los índices de dominancia y abundancia fueron mayores en época de estiaje. En el dendrograma de similaridad en base a los MIB se aprecia la formación de tres grupos en la época de avenida: un grupo respuesta a los pasivos mineros, un segundo grupo respuesta a impacto doméstico y agricultura, y un tercer grupo respuesta a impacto por la agricultura, industrias y doméstico. La calidad del agua fue determinada por los índices bióticos, EPT (principalmente mala sobre el 75\%) y el ABI (pésimomala: 16.67-33.33\%), mostrando que el río Rímac se encuentra seriamente afectado por diversas actividades antrópicas. Para los 
ensayos ecotoxicológicos con D. magna y con $C$. calligraphus solo la estación de muestreo $E_{1}$ fue tóxica en la época de avenida.

\section{Agradecimientos}

Se agradece a Ana Huamantinco, Christian Paredes, Ana Gutiérrez y Margarita Robles por las críticas al manuscrito. A Lizeth Cayo, Cosme Pascual, Olimpia Pariona, Hector Pascual y Alejandrina Cupa por el apoyo en materiales y colecta en campo para el desarrollo del presente trabajo.

\section{Literatura Citada}

1. Abarca FJ. 2007. Técnicas para evaluación y monitoreo del estado de humedales y otros ecosistemas acuáticos. En: Sánchez O, Mónica H, MárquezHuitzil PRE, Zambrano L. (eds). Perspectivas sobre conservación de ecosistemas acuáticos en México. México: Instituto Nacional de Ecología. p 113-144.

2. Acosta R, Ríos B, Rieradewall M, Prat N. 2009. Propuesta de un protocolo de evaluación de la calidad ecológica de ríos andinos (CERA) y su aplicación a dos cuencas en Ecuador y Perú. Limnetica 28: 35-64.

3. Alegre A, Bonifacio E, Lee S, Alvariño L, Iannacone J. 2018. Monitoreo ecotoxicológico de una cuenca en Huancavelica, Perú afectada por metales pesados. REDVET 19(5). [Internet]. Disponible en: http://www.veterinaria. org/revistas/redvet/n050518.html

4. Alomía J, Iannacone J, Alvariño L, Ventura K. 2017. Macroinvertebrados bentónicos para evaluar la calidad de las aguas de la cuenca alta del río Huallaga, Perú. Biologist 15: 65-84. doi: 10.24039/ rtb2017151144

5. Alonso A, Camargo JA. 2005. Estado actual y perspectivas en el empleo de la comunidad de macroinvertebrados bentónicos como indicadora del estado ecológico de los ecosistemas fluviales españoles. Ecosistemas 14: 87-89.
6. Alván AJ, Rodríguez CL, AlvánAguilar M. 2012. Evaluación preliminar de las comunidades de macroinvertebrados bentónicos de la laguna de Quistococha, Iquitos, Perú. Ciencia Amazónica 2: 86-92. doi: 10.22386/ ca.v2i2.30

7. Álvarez-Manzaneda E, RamosRodríguez E, López-Rodríguez MJ, Parra G, Funes A, De Vicente I. 2017. Acute and chronic effects of magnetic microparticles potentially used in lake restoration on Daphnia magna and Chironomus sp. J Hazard Mater 322: 437-444. doi: 10.1016/j.jhazmat.2016.10.035

8. Arambasic MB, Bjelic S, Subakov G. 1995. Acute toxicity of heavy: metals (copper, lead, zinc), phenol and sodium on Allium cepa $\mathrm{L}$, Lepidium sativum $\mathrm{L}$ and Daphnia magna St, comparative investigations and the practical applications. Water Res 29: 497-503. doi: 10.1016/0043-1354(94)00178-A

9. Arrascue A, Iannacone J, Alvariño L, Basilio S, Lazcano C. 2001. El insecto Chironomus calligraphus Goeldi y la bacteria Escherichia coli como ensayos ecotoxicológicos para evaluar sedimentos elutriados dulceacuícolas. Rev Per Ent 42: 159-173.

10. Bedregal P, Mendoza P, Ubillus M, Montoya E, Airas R, Baca L, Fajardo W. 2010. Evaluación de las aguas del río Rímac en Lima, Perú utilizando el índice de calidad de agua (ICA). Informe Científico. Lima: IPEN. p 13-19.

11. Burrial AT, Ocharán FJ. 2007. Comparación del muestreador de macroinvertebrados bentónicos fluviales con muestreador Surber y con red manual en río de Aragón (NE Península Ibérica). Limnética 26: 13-24.

12. Canteiro RCSA, Albertoni EF. 2011. Growth of immature Chironomus calligraphus (Diptera: Chironomidae) in laboratory conditions. An Acad Bras Cienc 83: 1279-1286. doi: 10.1590/ S0001-37652011005000035 
13. Carrera C, Fierro K. 2001. Manual de monitoreo. Los macroinvertebrados acuáticos como indicadores de la calidad del agua. Quito, Ecuador: Ed Eco Ciencia. $67 \mathrm{p}$.

14. Costa CR, Olivi P, Botta CMR, Espindola ELG 2008. A toxicidade em ambientes aquáticos: discussão e métodos de avaliação. Quím Nova 31: 18201830. doi: 10.1590/S0100-40422008000700038

15. Cózatl-Manzano R, Naranjo-García E. 2007. First records of freshwater molluscs from the ecological reserve El Edén, Quintana Roo, México. Rev Mex Biodivers 78: 303-310.

16. Darrigran G, Vilches A, Legarralde T, Damborenea C. 2007. Guía para el estudio de macroinvertebrados. I. Métodos de colecta y técnicas de fijación. Serie Técnica Didáctica N. ${ }^{\circ}$ 10. Probiota, FCNyM, UNLP. La Plata, Buenos Aires, Argentina. 35 p.

17. De Schamphelaere KA, Janssen CR. 2002. A biotic ligand model predicting acute copper toxicity for Daphnia magna: the effects of calcium, magnesium, sodium, potassium, and $\mathrm{pH}$. Environ Sci Technol 36: 48-54. doi: 10.1021/ es000253s

18. Domínguez E, Hubbard MD, Peters WL. 1992. Clave para ninfas y adultos de las familias y géneros de Ephemeroptera (Insecta) sudamericanos. Biol Acuát 16: 1-32.

19. Espinoza N, Morales FE. 2008. Macroinvertebrados bentónicos de la laguna Las Peonías, Estado de Zulia, Venezuela. Bol Cent Investig Biol 42: 345-363.

20. Fierro $P$, Bertrán $C$, Mercado $M$, Peña-Cortes F, Tapia J, Hauenstein E, Vargas-Chacoff L. 2012. Benthic macroinver-tebrate assemblages as indicators of water quality applying a modified biotic index in a spatio-seasonal context in a coastal basin of Southern Chile. Rev Biol Mar Oceanog 47: 2333. doi: 10.4067/S0718-19572012000100003
21. Figueiras FG Niell FX. 1986. Visibilidad del disco de Secchi, clorofila a y materia orgánica particulada en la ría de Pontevedra (NO de España). Invest Pesq 50: 607-637.

22. Figueroa $R$, Valdovinos $C$, Araya $E$, Parra O. 2003. Macroinvertebrados bentónicos como indicadores de calidad de agua de ríos del sur de Chile. Rev Chil Hist Nat 76: 275-285. doi: 10.4067/ S0716-078X2003000200012

23. Figueroa R, Palma A, Ruíz, V, Niell X. 2007. Análisis comparativo de índices bióticos utilizados en la evaluación de la calidad de las aguas en un río Mediterráneo de Chile: río Chillán, VIII Región. Rev Chil Hist Nat 80: 225-242. doi: 10.4067/S0716-078X2007000200008

24. Gonçalves FB, de Menezes MS. 2011. A comparative analysis of biotic indices that use macroinvertebrates to assess water quality in a coastal of Paraná state, southern Brazil. Biota Neotrop 11: 27-36. doi: 10.1590/S1676-06032011000-40000

25. Guerrero N. 2014. Contaminantes metálicos en sedimentos y suelos agrícolas generados por actividades mineras en la cuenca del rio Rímac. [Internet] Disponible en: http://www.bvsde.paho.org/ bvsaidis/ecuador10/neli.pdf

26. Guerrero-Bolaño F, ManjarresHernández A, Nuñez-Padilla N. 2003. Los macroinvertebrados bentónicos de Pozo Azul (cuenca del río Gaira, Colombia) y su relación con la calidad del agua. Acta Biol Colomb 8: 43-55.

27. Guillen GO, Cóndor EV, Gonzales TM, Iglesias LS. 1998. Contaminación de las aguas del río Rímac: trazas de metales. Rev Inst Investig Fac Minas Metal Cienc Geogr 1: 127-145.

28. Hammer $\emptyset$, Harper DAT, Ryan PD. 2005. PAST-Palaeontological Statistics, ver. 1.34 (2005). [Internet]. Disponible en: http://folk.uio.no/ohammer/past

29. Hynes HBN. 1970. The ecology of stream insects. Annu Rev Entomol 15: 25-42. doi: 15.010170.000325

30. Iannacone J, Alvariño L. 2007. Diversidad y abundancia de comunidades- 
zooplanctónicas litorales del humedal Pantanos de Villa, Lima, Perú. Gayana 71: 49-65. doi: 10.4067/S0717-65382007000100006

31. Iannacone JA, Dale WE. 1999. Protocolo de bioensayo ecotoxicológico para evaluar metales pesados contaminantes de agua dulce con Chironomus calligraphus (Diptera: Chironomidae) y el crustáceo Moina macrocopa (Crustácea: Cladócera), en el río Rímac. Rev Per Ent 41: 111-120.

32. Iannacone J, Salazar N. 2007. Efecto toxicológico de muestras de agua del Lago Junín, Perú, sobre Chironomus calligraphus (Diptera: Chironomidae). Braz J Toxicol 2: 219-227. doi: 10.5132/ jbse.2007.03.003

33. Iannacone J, Dale W, Alvariño L. 2000. Monitoreo ecotoxicológico del río Rímac (Lima-Perú) empleando a Chironomus calligraphus Goeldi (Diptera: Chironomidae). Rev Chil Ent 27: 25-34

34. Iannacone J, Mansilla J, Ventura K. 2003. Macroinvertebrados en las lagunas de Puerto Viejo, Lima, Perú. Ecol Apl 2: 116-124. doi: 10.21704/rea.v2i12.258

35. Iannacone J, Arrascue A, Cárdenas M. 2005. Evaluación de la ecotoxicidad de sedimentos del río Aruri y del Lago Junín (Perú) usando Escherichia coli y Chironomus calligraphus. Rev Bras Toxicol 18: 27-41.

36. Iannacone J, Alvariño L, Jimenez-Reyes, R, Argota G 2013. Diversity of plankton and macrozoobenthos as alternative indicator of water quality of Lurin River in the district of Cieneguilla, Lima-Peru. Biologist 11: 79-95.

37. Jin-Yong Ha, Masashi K, Masaki S. 2017. Acute toxicity of copper to Daphnia galeata under different magnesium and calcium conditions. Limnology 18: 63-70. doi: 10.1007/ s10201-016-0489-4
38. Llontop HC, Cabrera CC. 2010. Calidad del agua en la cuenca del río Rímac, sector de San Mateo, afectado por las actividades mineras. Rev Inst Investig Fac Minas Metal Cienc Geogr 13: 87-94.

39. Martella MB, Trumper EV, Bellis LM, Renison D, Giordano PF, Bazzano G, Gleiser RM. 2012. Manual de Ecología. Evaluación de la biodiversidad. Reduca (Biología). Serie Ecología 5: 71-115.

40. Manzo V. 2005. Key to the South America genera of Elmidae (Insecta: Coleoptera) with distributional data. Stud Neotrop Fauna E 40: 201-208. doi: 10.1080/01650520500140619

41. Méndez W. 2005. Contamination of Rimac river basin Peru, due to mining tailings. MSc Thesis. Sweden: KTH Royal Institute of Technology. $31 \mathrm{p}$.

42. Merritt RW, Cummins KM. 1996. An introduction to the aquatic insects of North America. $3^{\text {rd }}$ ed. USA: Kendall Hunt Publishing. 862 p.

43. [MINSA] Ministerio de Salud. 2010. Evaluación de los resultados de los monitoreos realizados a los recursos hídricos en la cuenca del río Rímac, en el marco del Convenio $\mathrm{N}^{\circ}$ 002-2009/ MINSA correspondiente al periodo de agosto a diciembre de 2009. Dirección General de Salud Ambiental. 56 p. [Internet]. Disponible en: http:// www.digesa.minsa.gob.pe/DEPA/rios/ $2009 /$ RIO_RIMAC_DIGESASEDAPAL_2009.pdf

44. Munkittrick KR, Power EA, Sergy GA. 1991. The relative sensitivity of microtox, daphnid, rainbow trout, and fathead minnow acute lethality test. Environ Toxicol Water 6: 35-62. doi: 10.1002/tox.2530060105

45. Muñoz-Riveaux $S$, Naranjo-López $C$, Gárces-González G, González-Lazo DD, Musle-Cordero Y, RodríguezMontoya L. 2003. Evaluación de la calidad del agua utilizando los macroinvertebrados bentónicos como bioindicadores. Rev Chapingo Ser CIE 9: 147-153. 
46. Oller C, Goitia E. 2005. Macroinvertebrados bentónicos y metales pesados en el río Pilcomayo (Tarija, Bolivia). Rev Bol Ecol 18: 17- 32.

47. Ortega H, Chocano, L, Palma C, Samanez I. 2010. Biota acuática en la Amazonía peruana: diversidad y usos como indicadores ambientales en el Bajo Urubamba (Cusco-Ucayali). Rev Peru Biol 17: 29-36. doi: 10.15381/rpb.v17i1.47

48. Oscoz J, Campos F, Escala MC. 2006. Variación de la comunidad de macroinvertebrados bentónicos en relación con la calidad de las aguas. Limnetica 25: 683-692.

49. Oyague-Passuni E. 2009. Discusión sobre el número de unidades muestrales y tamaño de unidad muestral para la estimación de valores de densidad de macroinvertebrados bentónicos en ambientes lóticos. Ecol Apl 8: 61-70. doi: 10.21704/rea.v8i1-2.383

50. Pan BZ, Wang HZ, Ban $X$, Yin $X A$. 2015. An exploratory analysis of ecological water requirements of macroinvertebrates in the Wuhan branch of the Yangtze River. Quatern Int 380-381: 256-261. doi: 10.1016/j.quaint.2014.10.011

51. Paredes C, Iannacone J, Alvariño L. 2004. Macroinvertebrados bentónicos como indicadores biológicos de la calidad de agua en dos ríos de Cajamarca y Amazonas, Perú. Rev Per Ent 44: 107-118.

52. Paredes C, Iannacone J, Alvariño L. 2005. Uso de macroinvertebrados bentónicos como bioindicadores de la calidad de agua en el río Rímac, LimaCallao, Perú. Rev Colomb Entomol 31: 219-225.

53. Pave PJ, Marchese M. 2005. Invertebrados bentónicos como indicadores de calidad del agua en ríos urbanos (Paraná-Entre Ríos, Argentina). Ecol Austral 15: 183-197.

54. Paz-y-Miño M, Barzola C, Lazcano C, Ponce M, León J. 2003. Colifagos como indicadores de contaminación fecal y de remoción bacteriana en la potabilización del agua. Rev Peru Biol 10: 133-144. doi: 10.15381/rpb.v10i2.2495

55. Rico C, Paredes M, Fernández $N$. 2009. Modelación de la estructura jerárquica de macroinvertebrados bentónicos a través de redes neuronales artificiales. Acta Biol Colomb 14: 71-96.

56. Righi-Cavallaro KO, Roche $\mathrm{KF}$, Froehlich O, Cavallaro MR. 2010. Structure of macroinvertebrate communities in riffles of a Neotropical karst stream in the wet and dry seasons. Acta Limnol Bras 222: 306-316. doi: 10.4322/ actalb.02203007

57. Ríos-Touma B, Encalada AC, Prat FN. 2011. Macroinvertebrate assemblages of an Andean high-altitude tropical stream: the importance of season and flow. Int Rev Hydrobiol 96: 667-685. doi: 10.1002/iroh.201111342

58. Rivera H, Chira J, Zambrano K, Petersen P. 2007. Dispersión secundaria de los metales pesados en sedimentos de los ríos Chillón, Rímac y Lurín Departamento de Lima. Rev Inst Investig Fac Minas Metal Cienc Geogr 20: 19-25.

59. Rodríguez-Varela AC, Fonseca-Romero MA, Vázquez-López H, CruzGómez A. 2018. Determinación de la calidad del agua mediante indicadores biológicos en la presa Himoay, municipio de Ilotepec, estado de México, México. Biologist 16: 159-170. doi: 10.24039/ rtb2018161228

60. Roldán PG. 1988. Guía para el estudio de los macroinvertebrados acuáticos del departamento de Antioquia. COLCIENCIAS. Universidad de Antioquia. Medellín. [Internet]. Disponible en: https://www.ianas.org/docs/books/ wbp13.pdf

61. Roldán PG. 1992. Fundamentos de limnología neotropical. Medellín, Colombia: Ed Univ. de Antioquia. 529 p.

62. Roldán PG 2003. Bioindicación de la calidad del agua en Colombia. Uso del método BMWP/Col. Antioquía, Colombia: Ed Univ. de Antioquía. 170 p. 
63. Román-Farje A, Iannacone J, Alvariño L. 2017. Efecto tóxico del saúco, Sambucus peruviana (Caprifoliaceae), en Daphnia magna, Sitophilus zeamais y Copidosoma koehleri en Peru. Chil J Agric Anim Sci 33: 3-13. doi: 10.4067/S0719-38902017005000101

64. Rosemberg D, Resh V. 1993. Freshwater biomonitoring and benthic macroinvertebrate. New York, USA: Chapman and Hall. 488 p.

65. Souto RM, Facure K, Pavanin L, Jacobucci G. 2011. Influence of environmental factors on benthic macroinvertebrate communities of urban streams in Vereda habitats, Central Brazil. Acta Limnol Bras 23: 293-306. doi: 10.1590/S2179-975X2012005000008

66. Trama FA, Rizo Patrón FL, Springer M. 2009. Macroinvertebrados bentónicos del humedal de Palo Verde, Costa Rica. Rev Biol Trop 57: 275-284.

67. [SETAC] Society of Environmental Toxicology and Chemistry. 2004. Whole effluent toxicity testing. [Internet].
Available in: https://cdn.ymaws.com/ www.setac.org/resource/resmgr/publications_and_resources/tip-wet.pdf

68. [USEPA] United States - Environmental Protection Agency. 2000.

Method guidance and recommendation for whole effluent toxicity (WET) Testing (40CFR Part 136). [Internet]. Available in: https://nepis.epa.gov/Exe/tiff2png.cgi/ P 10099 B C. P N G ? - r + 75 + $\mathrm{g}+7+\mathrm{D} \% 3 \mathrm{~A} \% 5 \mathrm{CZYFILES} \% 5 \mathrm{CIND}-$ EX\% 20DATA\% 5C00THRU05\% 5CTIFF\%5C00001619\%5CP10099BC.TIF

69. Villamarín C, Rieradevall M, Paul MJ, Barbour MT, Prat N. 2013. A tool to assess the ecological condition of tropical high Andean streams in Ecuador and Peru: The IMEERA index. Ecol Indic 29: 79-92. doi: 10.1016/j.ecolind.2012.12.006

70. Zilli FL, Montalto L, Paggi AC, Marchesem MR. 2008. Biometry and life cycle of Chironomus calligraphus Goeldi 1905 (Diptera, Chironomidae) in laboratory conditions. Interciencia 33: 767- 770. 